\title{
From Scale to Information and Back: Human Social Evolution in the Holocene
}

\author{
Jaeweon Shin ${ }^{1}$, Michael Holton Price ${ }^{2}$, David H. Wolpert ${ }^{2,3, *}$, Hajime Shimao ${ }^{2}$, Brendan \\ Tracey $^{2}$, and Timothy A. Kohler ${ }^{2,4,5,6, *}$
}

\author{
${ }^{1}$ Rice University, \\ ${ }^{2}$ Santa Fe Institute, \\ ${ }^{3}$ Arizona State University, \\ ${ }^{4}$ Washington State University, \\ ${ }^{5}$ Crow Canyon Archaeological Center, \\ ${ }^{6}$ Research Institute for Humanity and Nature
}

\section{ABSTRACT}

We use the recently introduced Seshat database to investigate the long-timescale development of human societies. Seshat contains high-dimensional sociopolitical features for hundreds of polities, from multiple continents, over many thousands of years. Examining the statistical covariations among those social features, we find that the process of sociopolitical development is dominated first by growth in polity scale, then by improvements in its information processing and economic systems, and then by further increases in scale. This allows us to define a Scale Threshold for societies, beyond which growth in information processing becomes paramount, and an Information Threshold, which once crossed permits additional growth in scale. Polities diverge from one another in sociopolitical feature space prior to crossing the Information Threshold, but then reconverge. Our results have implications for the timing of the appearance of moralizing gods, the role of population growth and institutions in sociopolitical evolution, and the causes for the evolutionary divergence between Old and New World polities.

At any single moment over the last 10,000 years, there has been great variation in how the human societies of the world are organized. Despite this cross-sectional heterogeneity, there appear to be striking regularities governing the evolution of societies through time. The domestication of plants and animals and formation of sedentary agricultural villages developed independently in societies in about a dozen separate regions, in both the Old and New Worlds, following climatic stabilization at the end of the last Ice Age ${ }^{1-3}$. In many of these cases, domestication of plants and animals combined with changing human mobility patterns to generate rapid population growth ${ }^{4,5}$. Rather similar processes of urbanization eventually followed this population growth in many parts of both the Old and New Worlds ${ }^{6-8}$. In most societies, wealth inequality increased with greater reliance on food production, with the development of technologies yielding more surplus, with more efficient wealth transmission between generations, and with more prominent political hierarchies and various other factors promoting economic defensibility ${ }^{9-11}$.

More quantitatively, there is pronounced covariation among the values of many social variables when assessed over long periods. Productive agricultural systems, large group sizes, urbanism, political hierarchy and high levels of wealth inequality often - though not completely - coincide. In every region of the world permitting agriculture, we find considerable similarity in both the beginning forms of human social groups, as relatively small-scale hunter-gatherer groups, and their ultimate (or at least current) forms, as large-scale urban societies. All the interesting variability — the history — lies in the differing ways that the societies in each region develop from those beginnings.

To determine whether these shared patterns in the dynamics of societies through time reflect propinquity 
and borrowing, shared roots, or entrainment along common paths via competitive pressures requires detailed data on their development around the world, stretching back at least to the end of the Pleistocene. Fortunately, our knowledge of Holocene prehistory and history has expanded rapidly in the last several decades, facilitated by the requirement in many countries for site investigation prior to destruction by development ${ }^{12}$, by increased chronological accuracy (e.g., ${ }^{13}$ ), and by improvements in the scope and precision of various climatic proxies that permit better understanding of the role of climatic variability in sociopolitical change ${ }^{14}$. Due to the rapid accumulation of such data, once-authoritative comparative studies (e.g., ${ }^{15,16}$ ) deserve continuous re-evaluation, especially since these earlier studies emphasized the more recent, historical end of the spectrum of increasing sociopolitical complexity.

One notable project addressing this demand has resulted in a dataset called "Seshat: Global History Databank"17. This is an ambitious coding of more than 1500 variables containing state-of-the-art knowledge on variables related to social complexity for hundreds of polities. The polities in the Seshat database for which comprehensive data are available span six continents and date from the Neolithic to the middle of the last millennium.

This dataset has enabled a number of recent publications ${ }^{17-21}$. Particularly important here, Peter Turchin and colleagues ${ }^{22}$ combined 51 variables recorded in Seshat to produce nine new variables, which they call "complexity characteristics" (CCs). They then performed a principal component analysis (PCA) of the 285 polities in that nine-dimensional CC space, as described in more detail below and in the SI. They found that the first principal component (PC / PC1) captures $77 \%$ of the underlying variation in the dataset. In addition, they found that polities almost always increase their PC1 value with time. This led them to portray sociopolitical evolution as a largely homogeneous process through time in which scalar growth (in population, capital population, and territory size) and increase in the number of levels and sophistication of government, infrastructure, writing, texts, and exchange media all occur in a highly correlated fashion.

The PCA of Turchin and colleagues answers the question, "what is the largest dimension of shared variability across all the variables and among all the cases in the Seshat data?", where these cases range from village-level societies to empires. It is important to note that this question is essentially ahistorical, disembedding the cases (a particular polity in a particular century) from their regions and their own trajectories of development. In contrast, here we are more interested in questions like "how do polities become more complex?" or "what paths do they follow through the Seshat feature space?" The fact that the first principal component of a PCA by itself captures a large amount of the variation in the data does not preclude there being rich structure relating all of the components. To give a stark example, suppose that we have a two-dimensional dataset, generated by sampling an underlying dynamics of the form $x(t)=t, y(t)=\sin (t)$. If the data comes from a long enough range of $t$ values, then a PCA will tell us that PC1 is (approximately) identical to the $x$ direction, while PC2 is identical to the $y$ direction, and that PC1 "captures a large amount of the variation in the data". Yet in fact there is more to understand about the relationship between PC1 and PC2 (Figure 1), namely $y=\sin (x){ }^{1}$

Motivated by such considerations, in this article we analyze the covariation between the values of the top two principal components in the Seshat dataset. We find that there is a striking, highly patterned relationship between them - similar to the sine wave relationship above. To help understand the social and historical significance of this relationship, we examined the weightings (loadings) of the CCs under

\footnotetext{
${ }^{1}$ Ironically, the fundamental issue we raise is very closely related to one identified by Turchin and Korotayev who noted that correlational analyses of cross-cultural data yield misleading results when two variables (population density and incidence of warfare, in their case) are linked in a dynamical system and oscillate relative to each other with time lags, as in a predator-prey system $^{23}$. In the case of the correlational analysis $\mathrm{in}^{22}$ the issue is not with time lags, though, but with changes in the direction of the relationship between PC1 and PC2 across the developmental ranges of the societies they describe.
} 
the vector PC1 and under the vector PC2. This provides our first result: According to the Seshat database, the development of polities on average is dominated first by a period of growth in scale, e.g., the capital's population and territory size. After that the dynamics enters a period when it is dominated by improvements in information-processing and economic systems (i.e., by transactional and informationstorage capabilities). These improvements are then followed by further increases in scale in a third period. This result suggests that to make major improvements in information processing, a polity must first reach a threshold in scale. It also implies that the further increases in scale in the third region depend in turn on improvements in information-processing ability. Social evolution is thus a contingent, historical process.

Next we investigate the dynamics of the regions recorded in the Seshat dataset, making full use of the century-resolution time-stamps pro-

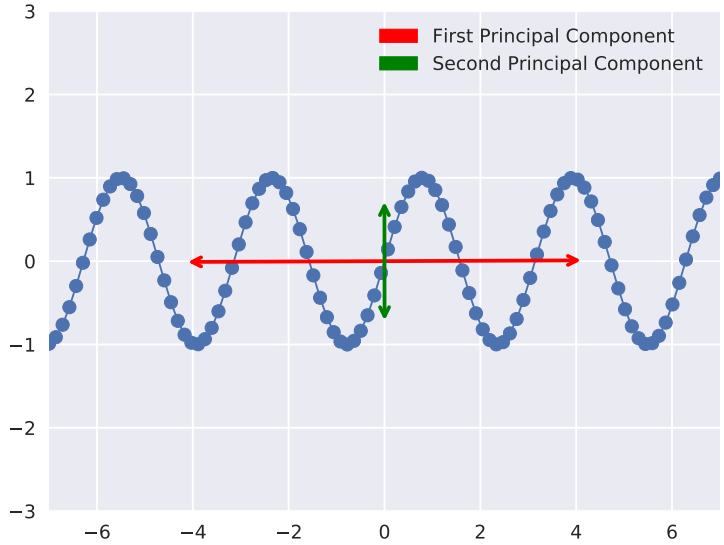

Figure 1. PCA decomposition of the data generated from sinusoidal function. Data points on the plot are drawn at uniform interval from parametric equations $x(t)=t$ and $y(t)=\sin (2 t)$, where $t$ ranges from -7 to 7. The red bidirectional line represents the first Principal Component, which explains $98 \%$ of the variance in the data. The green bidirectional line represents the second Principal Component, which explains the rest of the variance in the data vided in Seshat. This provides our second, albeit qualitative result: There appears to be a first region in PC1-PC2 space in which polities are relatively concentrated. This is followed by a second region where polities become more spread apart; we can speak of them taking different paths in their growth in scale. In a final, third region, there appears to be a pronounced homogenization of features in which polities come to lie almost on top of one another. We note that this final convergence might simply reflect saturation of the values of the particular features that are recorded in Seshat, which were chosen with pre-modern polities in mind. Intriguingly, the first region coincides with the scale-dominated growth described above. The second region coincides with a more variable experience where many but not all polities achieve improvements in information-processing broadly conceived. The third region coincides with renewed emphasis on growth in scale.

We interpret the two borders between these three regions as social complexity boundaries. The first, which we call the Scale Threshold, separates polities that have undergone more growth in scale than in information-processing capacity, from those which will be differentially expanding their information-processing capacity. The second, which we call the Information Threshold, separates polities that have for the most part achieved the processing capacity for additional growth in scale, from those which have fully exploited that capacity through increases in scale. We can use this interpretation to analyze other datasets so long as we can calculate the (Seshat) PC1 values of those polities. We illustrate this to augment a recent analysis ${ }^{21}$ as to whether moralizing gods appear in a polity before or after it crosses the second of these thresholds.

While performing these analyses we uncovered patterns which turned out to be statistical artifacts. In particular, there is a pronounced clustering of the PC1 values in Seshat in two distinct regions, which can be accurately described as a mixture of two Gaussians of very similar widths. Such clusters in a sociopolitical feature space arise often in archaeological datasets, and are sometimes interpreted as "basins 
of attraction" of an underlying dynamics ${ }^{24}$. However, as we discuss in the SI, the clustering in Seshat appears to be an artifact of a type not so far described in the literature, to our knowledge. This provides a cautionary tale on analyzing datasets that combine samples of multiple, finite duration runs of the same Markov chain, if (as in Seshat) each run has a different starting position and a different starting time.

The SI contains more details on the Seshat database and our analyses of it.

\section{Results}

\section{Interaction between PC1 and PC2}

We find a pronounced structure relating the values of PC1 with the values of the other eight principal components, despite PC1 containing $77 \%$ of the covariation across polity feature vectors. Here we report the relationship between the first and second principal components, PC1 and PC2. Figure 2 shows the change in the average of polity scores ${ }^{2}$ on PC2 in a sliding window along PC1 space. It is clear that a non-linear interaction between PC1 and PC2 exists, where the average PC2 scores of polities first drop, then rise, and then drop again along PC1. In other words, there is an interdependent relation between PC1 and PC2 despite no Pearson correlation in aggregate.

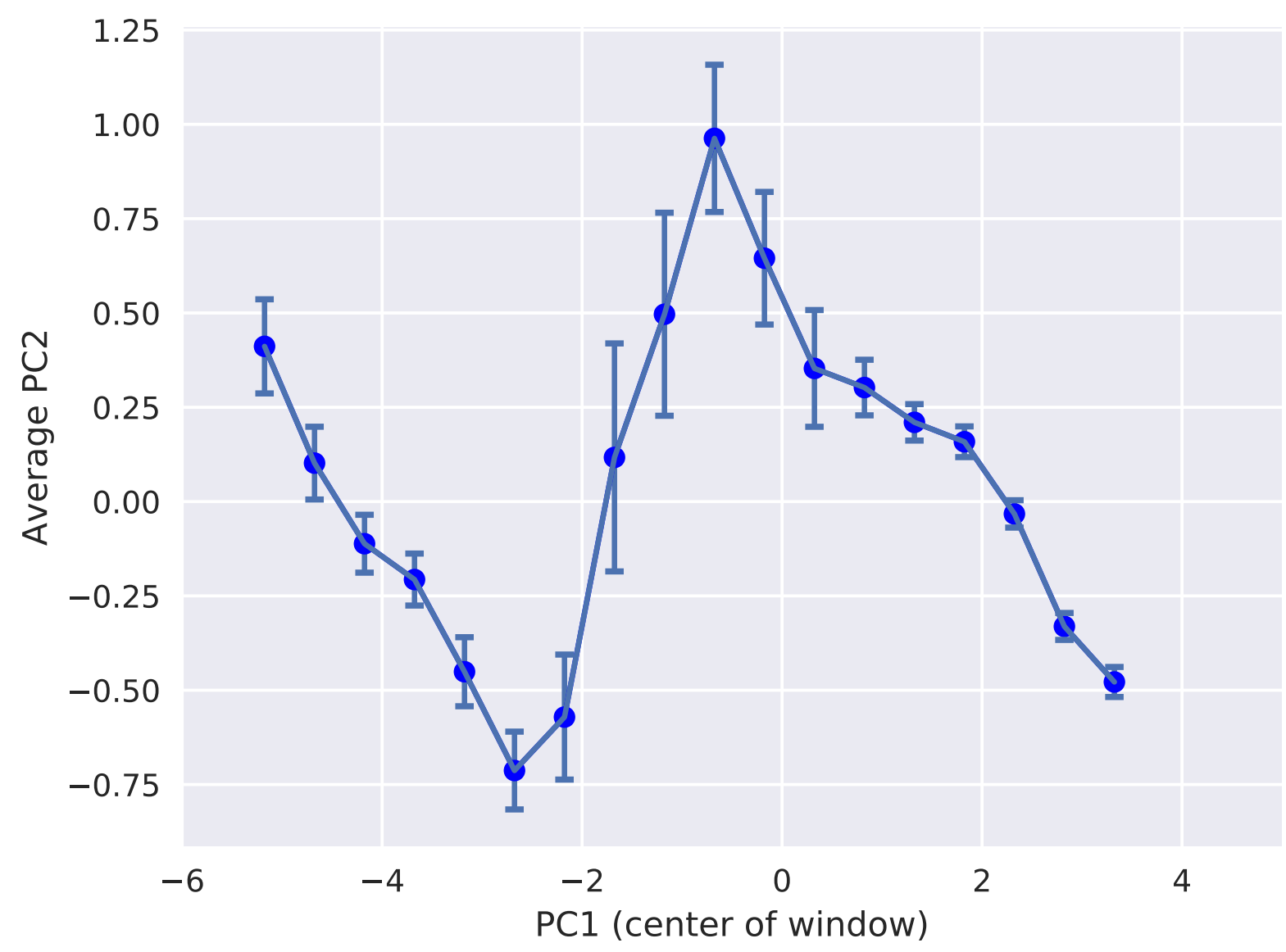

Figure 2. The average score of observations on PC2 in a sliding window along PC1.

\footnotetext{
2 the projection of a unit on a vector
} 
It is important to be clear about how the changes in direction in Figure 2 should be interpreted. Movement to the right along the PC1 axis happens as polities have increasing values for those CCs that have positive loadings on PC1 in Table 1 - that is, all the CCs, though led most strongly by PolPop, texts, and CapPop. Movement downwards on the PC2 axis indicates increasing values on those CCs that are negatively correlated with PC2 - led by PolTerr, CapPop, and PolPop - while values on CCs with positive loadings on PC2 (led by money and writing) increase more slowly or not at all. On the other hand, movement upwards along PC2 happens when values for those CCs with positive loadings on PC2 are differentially increasing. Once again these are money, writing, and texts. Together these three CCs (and less importantly those with lower positive scores on PC2 in Table 1) reflect what we call the information-processing capacity of the polity.

The first "hinge point" in Figure 2 occurs near the PC1 value of -2.5 where average PC2 values abruptly stop decreasing and start to increase. Polities located near this point include Upper Egypt ca. 3600 BC, the Paris Basin ca. 400 BC (La Tène A), the Iceland Commonwealth ca. AD 1000, and the Valley of Oaxaca at the same time. The second hinge point in Figure 2 occurs at a value for PC1 of around -0.5, where average values of PC2 suddenly switch from increasing to decreasing as PC1 increases. Polities located near this hinge include proto-Elamite Susiana ca. 3000 BC, and First Intermediate period Upper Egypt ca. 2100 BC.

\begin{tabular}{|l|c|c|c|c|c|c|c|c|c|c|}
\hline & PolPop & PolTerr & CapPop & levels & gvrnmt & infrastr & writing & texts & money & \%Var \\
\hline PC1 & 0.95 & 0.87 & 0.91 & 0.89 & 0.88 & 0.88 & 0.86 & 0.92 & 0.79 & 77.2 \\
PC2 & -0.23 & -0.30 & -0.27 & -0.21 & 0.08 & 0.09 & 0.33 & 0.23 & 0.34 & 6.0 \\
\hline
\end{tabular}

Table 1. Pairwise correlations between the nine Complexity Components and PC1 and PC2, and the percentage of variance explained by each PC. Derived in part from [22, Table SI5].

Figure 3 decomposes PC2 into two sets of CCs, ones with positive loadings on PC2 (money, writing, texts, infrastructure and gvrnmt) and others with negative loadings (PolTerr, CapPop, PolPop and levels). Again, the CCs with positive PC2 loadings are related to information-processing capabilities, while those with negative loadings are related to scale. For each PC1 value, the green and blue lines represent the contribution of the two sets of CCs to PC2 (the sum of the product of the CC values and their loading on PC2). In other words, the green (blue) line indicates the PC2 value evaluated only using the information(scale-) related CCs. The red line is the summation of the green and blue, and is equivalent to the line in Figure 2.

As polities grow in their PC1 value, the relative contributions of the scale CCs and the informationprocessing CCs changes. Up to the first hinge point at PC1 $\sim-2.5$, polities grow substantially in scale as their PC1 value increases (i.e., the blue line drops), with relatively less growth in their informationprocessing capabilities. Since the PC2 vector has negative components for those scale-related CCs, this results in declining values on PC2. Between -2.5 and -0.5 on PC1, movement along PC1 is accompanied by relatively rapid increases in information-processing capabilities (though the error bars indicate more heterogeneity in scores on PC2 in this portion of PC1 than in any other portion). Since the PC2 vector has positive components for the information-processing CCs, this results in an increasing value of PC2 as PC1 grows in this region. Then as polities move past -0.5 in PC1, again growth in scale CCs dominates the change in information-processing CCs, resulting in the decreasing values of PC2 in Figure 2.

These dynamics suggest the possibility that at some point ${ }^{3}$ growth in scale may become constrained in polities where writing or money are relatively unsophisticated. Developing increased capacities in

\footnotetext{
${ }^{3}$ Specifically at a level of complexity represented by values of PC1 of about -2.5
} 


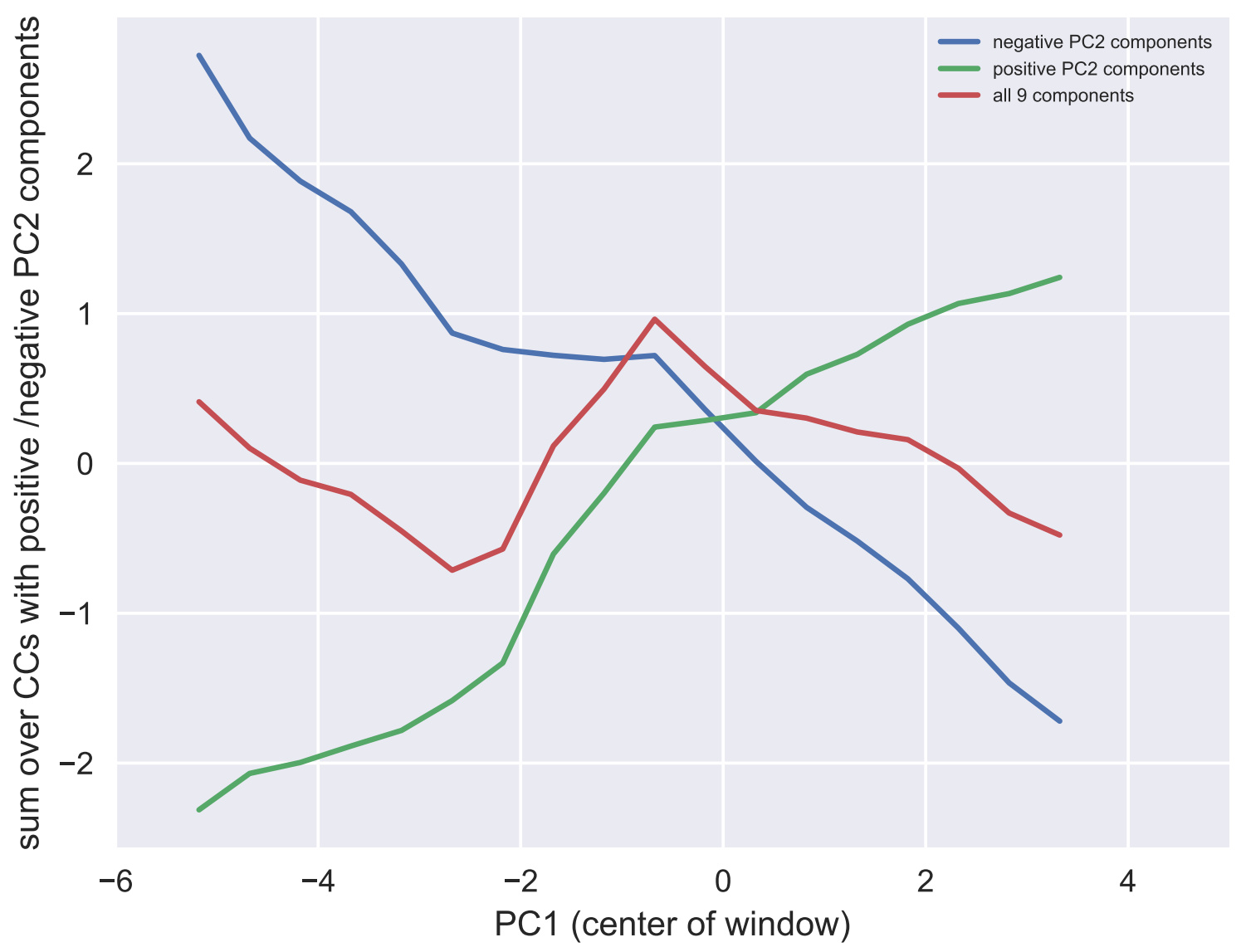

Figure 3. The decomposition of the average PC2 into two subsets of CCs. The green line represents the CC components related to information-processing capabilities (money, writing, texts, infrastructure and gvrnmt in order of importance), having positive correlations with PC2. The blue line represents the CC components primarily related to scale (PolTerr, CapPop, PolPop and levels in order of importance). The red line in this figure is identical to the blue line in Figure 2, with a change of scale.

these areas appears to allow further increases in scale. This is why we suggest that that hinge point can be interpreted as a "Scale Threshold", marking an important transition in the dynamics of developing polities. At PC1 values of about -0.5 another, "Information Threshold," is passed, appearing to allow further increases in scale.

\section{Dynamics in the PC1-PC2 space}

A priori, it is possible that the results in Figures 2 and 3 could be driven by a few aberrant cases, perhaps a few large societies of the Americas that lack writing or some small societies that have writing for some reason like borrowing, or because they have budded off from a larger society ${ }^{4}$. To help address this issue we now show that the movement upward in the PC2 dimension between values of about -2.5 to near 0 on

\footnotetext{
${ }^{4}$ All these possibilities were raised by a reviewer.
} 
PC1 is nearly universal in the Old World regions analyzed in $^{22}$, though not in the Americas ${ }^{5}$.

Figure 4 presents a more fine-grained picture of the Seshat PC1-PC2 values, by including the timestamps of the datapoints to depict trajectories of individual polities. Arrows run from an entry in Seshat giving the (PC1, PC2) coordinates of a particular polity at a particular time to the (PC1, PC2) coordinates of the next recorded time for that same polity. Typically, the time between such datapoints is 100 years, but often there are gaps in the sequence ${ }^{22}$. For observations more than 100 years apart, the dots in Figure 4 show the interpolated locations along each arrow at a spacing of 100 years, given a linear interpolation between the points $\mathbf{x}_{i}$ and $\mathbf{x}_{i+1}$. Red arrows stand for New World polities, and blue for those in the Old World.

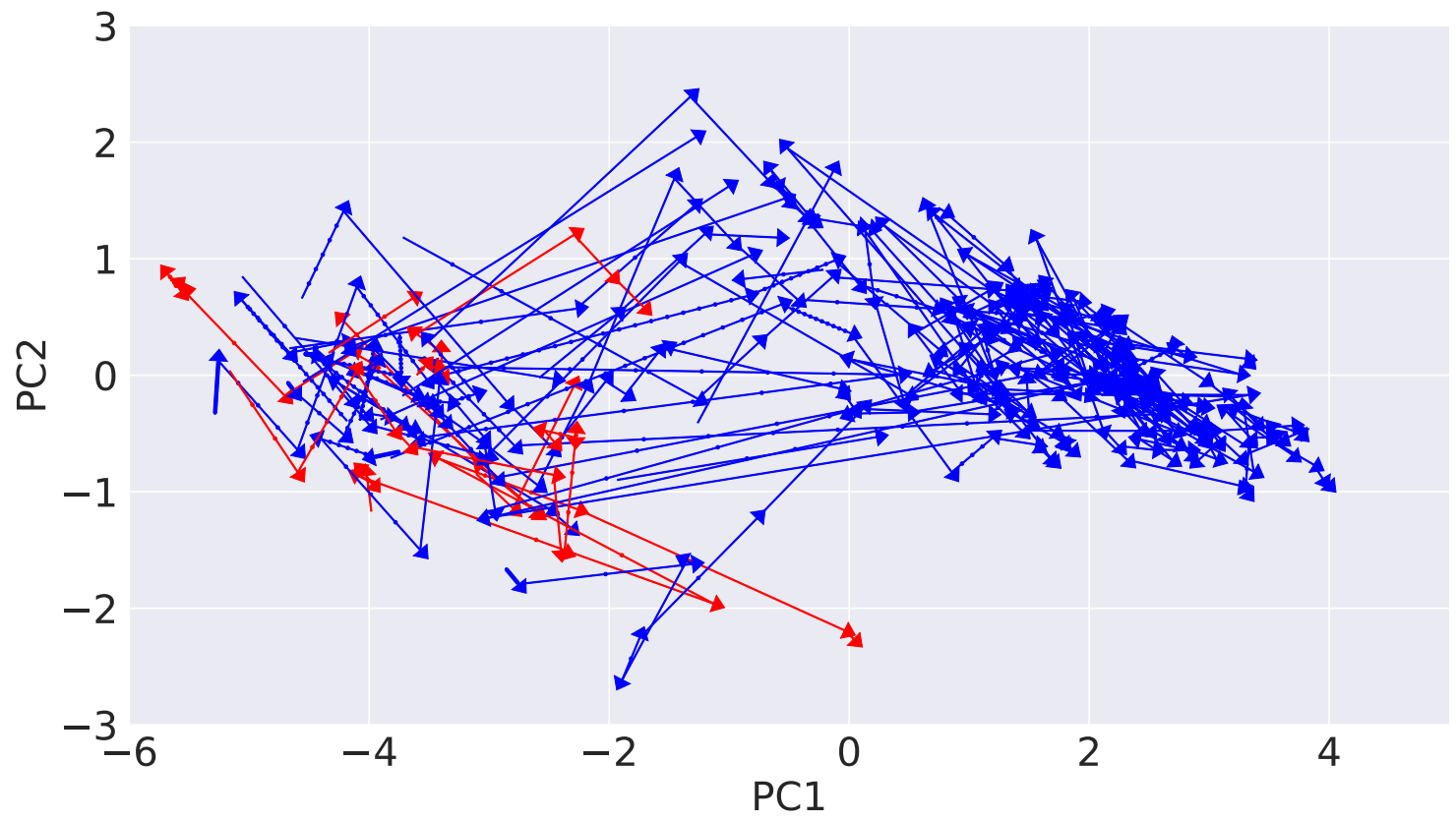

Figure 4. The flow of the original data in PC1-PC2 space. Red indicates New World NGAs; blue indicates Old World NGAs.

Figure 4 suggests several features of sociopolitical evolution that have been previously poorly recognized (if at all). First, the regions picked out by the hinge points in Figure 2 approximately coincide with other changes in the dynamics through PC1-PC2 space. For example, societies lying between the Scale and the Information thresholds are more heterogeneous on both dimensions than are societies lying to the right of the Information Threshold. Movement into the right-most region is in part a process of homogenization of features. Second, there are interesting dynamical patterns in all three regions. These patterns are primarily due to movement along the PC2 dimension, even though it is orthogonal to PC1 in aggregate. Although there is a general movement towards the right in all regions, there is quite a bit of variety in the direction of movement in the PC2 dimension: both positive and negative movements can be seen. Transitions from the first to the second region are fairly common and often involve large jumps, whereas transitions from the second region to the first are relatively rare but can also involve large jumps.

\footnotetext{
${ }^{5}$ The six NGAs analyzed in the New World are (North America) Finger Lakes, Cahokia, Valley of Oaxaca; (South America) Lowland Andes, North Colombia, and Cuzco. The complete sample is listed in Turchin et al. [22, Table SI1].
} 
Perhaps the most remarkable feature in Figure 4 though is the nearly universal trajectory upwards in the PC2 dimension in the middle of the PC1 range. This is what is causing the change in direction of the line in Figure 2. To reiterate the point made above, this reflects differential improvements in information processing in the middle zone of PC1 than in either of the other two zones. Note however the different trajectories of most New World societies in this region.

The general downwards slope of most trajectories for positive values of PC1 is noteworthy. It is tempting to conclude from informal examination of the plot, and its dense cluster of points around $(\mathrm{PC} 1=2, \mathrm{PC} 2=0)$ that there is a sink in the middle of the right cluster. While possible, this should be confirmed with further statistical analysis. It seems possible that this feature is related to saturation in the $\mathrm{CC}$ values, which were designed for characterizing pre-modern polities.

A final point of interest arising out of Figure 4 is that human societies do not exist in most of the possible space of joint social and cultural traits. The generally later cluster is even more focused on a portion of the available space than are the societies in the generally earlier cluster. We do not (for example) see societies with sophisticated information processing but small size. Those in the New World are largely confined to the left-hand cluster. Cahokia, for example, achieves its highest score on PC1 at A.D. 1200 $(-2.57$; PC2=-1.21), approaching but not quite crossing the Scale Threshold. Only the Cuzco NGA under the Inca in this sample crosses the Information Threshold, during the AD 1400s and 1500s, with a PC1 score of 0.02 in A.D. 1500, though markedly low scores on PC2 (-2.26 in A.D. 1500). Societies in the New World sample may be constrained in size by their low information-processing capacities.

Peregrine $^{25}$ reports an analysis of the archaeological traditions coded $\mathrm{in}^{24}$ in which factor analysis was used to define a two-dimensional "morphospace" within which each of the traditions could be located at a specific time. As in our analysis of Seshat, a great deal of the available morphospace was never (or

rarely) occupied. Following ${ }^{26}$, Peregrine attributes some of this empty space to functional constraints (for example, were small-scale societies to undertake construction of large-scale public monuments, it could only be to the [lethal] detriment of their day-to-day food-getting activities). Other empty space is likely due to developmental constraints (for example, small-scale societies may simply not have enough interacting minds to innovate and maintain complex technologies).

The observation that societies focus on a smaller portion of the possible space defined by PCs 1 and 2 as they move into the "late" cluster is reminiscent of the common observation that small-scale societies are relatively more diverse than large-scale societies in terms of both cultural practices and organization (e.g., [27, pp. 8-10]). Note that in the part of the transition region where scores for PC1 hover around 0, polities with low scores on PC2 tend to move up, while polities with high values on the second PC tend to move down (Figure 4).

A plausible mechanism for explaining this convergence that deserves further examination is that the selective environments inhabited by societies become increasingly competitive as they move to the right along the PC1 dimension. Highly competitive environments reward societies with constellations of traits that allow them to survive and spread. Diverse societies either develop similar constellations of traits through this selection for what works best in this competitive environment, or they fail to survive. Similar dynamics are predicted in ${ }^{28,29}$.

\section{Appearance of Moralizing High Gods}

The social complexity thresholds uncovered here are broadly applicable and can be used to inform any analysis of a dataset that involves estimating when a given NGA makes a leap in social complexity. As an illustration, in this section we use these concepts to elaborate on a recently published analysis of the hypothesis that "belief in morally concerned supernatural agents culturally evolved to facilitate cooperation among strangers in large-scale societies"21. 
The analysis by Whitehouse and colleagues ${ }^{21}$ begins by introducing a new version of Seshat, augmented with variables concerning Belief in a Moralizing God (BMG) in each NGA through time. The authors then performed a time-series analysis of the trajectories of the NGAs in this dataset to assess whether they take a leap in social complexity before or after the appearance of BMG. (See the SI for a discussion of these new variables and other aspects of this analysis.)

This approach requires them to exclude any NGA without PC1 values both before and after BMG, since in such cases they could not assess whether a leap in social complexity occurred before or after BMG. This led them to consider only 12 NGAs while excluding 18 NGAs (7 of which have BMG always present). Based on this subsample, Whitehouse and colleagues ${ }^{21}$ concluded that moralizing high gods do not arise before societies become states.

Note that these NGAs were excluded because the method relied on a time-series analysis of each NGA to assess whether and when a leap in social complexity occurred. However, all datapoints in the full dataset - including NGAs excluded from ${ }^{21}$ - have known associated PC1 values. Instead of using time-series, we can simply observe whether the PC1 value of an NGA at that time is less than the Information Threshold.

This augmented BMG dataset is illustrated in Figure 5. This figure is a modification of Figure 4, where arrows are now colored by the moralising gods status of the datapoints at the base of each arrow, with grey for missing, blue for absent, and green for present. The red and orange dots mark the first appearance of moralising gods in any sequence. The red dots mark the 12 NGAs used by ${ }^{21}$ whereas the orange dots mark the 7 NGAs that they excluded even though they had at least one Present observation (not including Big Island Hawaii), all of which exhibit the pattern Always Present.

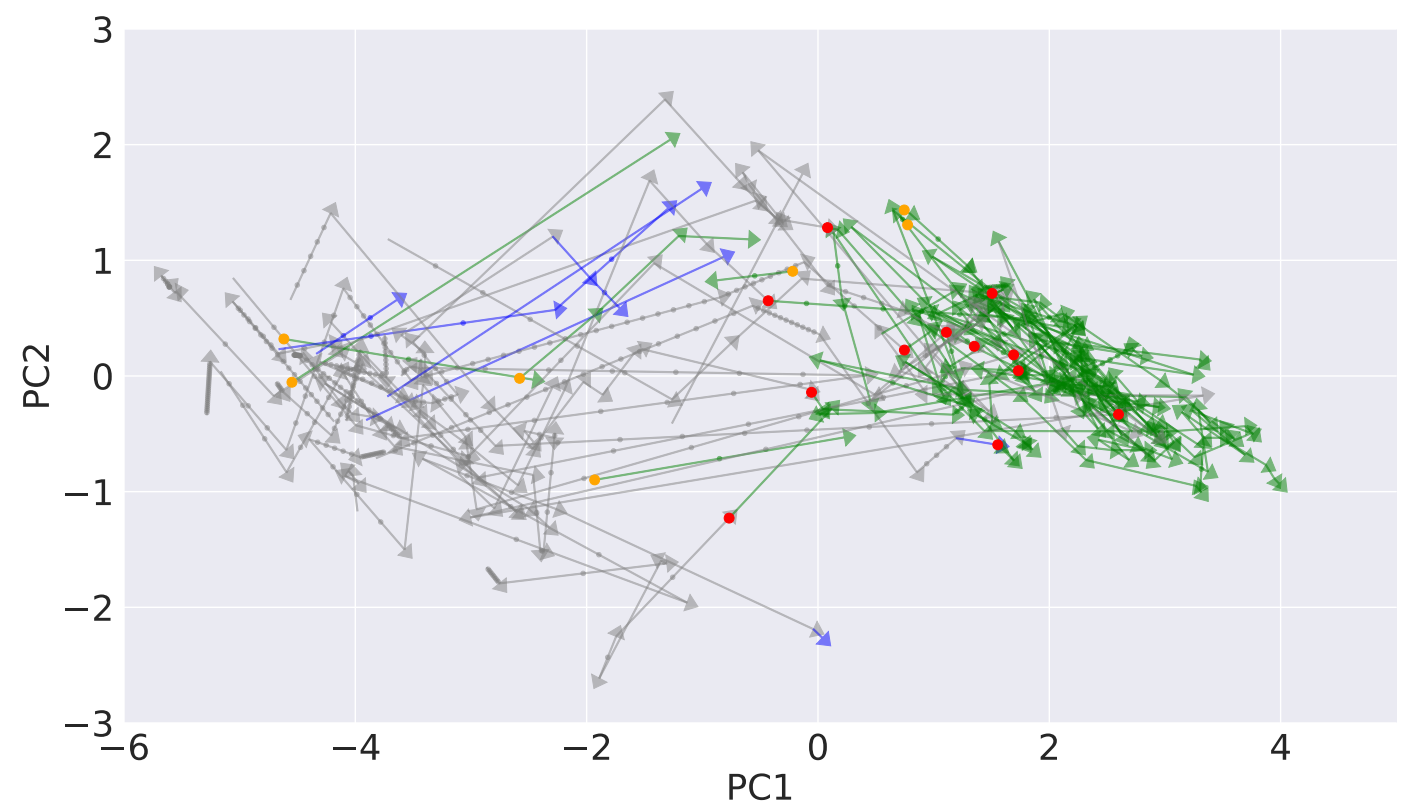

Figure 5. PC1-PC2 movement plot colored by moralizing gods status: NA / missing (grey), Absent (blue), and Present (green). For each NGA the dots mark the lowest PC1 value where the status becomes Present (regardless of the preceding value). Red dots mark the NGAs included by ${ }^{21}$; orange dots mark the NGAs they excluded.

Figure 5 suggests that the distribution of PC1 values at which Moralising Gods are first Present in an NGA does not differ substantially from the scatter of PC1 values themselves. The clustering of the red 
dots in or near the right cluster, which effectively creates the pattern noted by ${ }^{21}$, appears to result from the choice to analyze only a subset of the original 30 NGAs. Further, the assumption that Missing should be treated as Absent for 11 of those 12 NGAs, as assumed in ${ }^{21}$, would have the effect of shifting the red dots to the left in Figure 5, further weakening the claim in ${ }^{21}$ that complex societies precede moralizing gods throughout world history.

\section{Discussion}

To a first approximation, the Seshat database analyzed here and by ${ }^{22}$ demonstrates a shared movement through time by all the polities in this sample along an axis (PC1) representing simultaneous increases in scale and in a number of indicators of governmental, infrastructural, informational, and economic capacities. This homogeneous process is characterized as having produced a "striking similarity in the way that the societies in our global historical sample are organized" ( [20, p. 4 of 8]; see also [22, p. 3]).

Going beyond this first approximation however we demonstrate a striking structure relating the scale and information components recorded in Seshat. This structure strongly suggests that sociopolitical evolution is initiated primarily by growth in scale. Only once a certain size is reached, which we call the Scale Threshold, does growth in information-processing capacity start to become desirable. Eventually a polity's growth in scale may become limited by low capacity in its writing, exchange and similar "information-processing" systems. Once those capacities reach a certain level, polities can cross what we call the Information Threshold allowing further growth in scale.

Moreover, the leftmost zone in Figures 2 - 5 seems to be one in which different polities develop in idiosyncratic ways, diverging in the space of sociopolitical features. The second stage, dominated by growth in information-processing capabilities, seems to be one in which polities — with the notable exception of those in the New World - all start moving in parallel. Then in the third stage - across the Information Threshold - polities converge in feature space. We must caution of course since these patterns are all based on the current Seshat database which, impressive as it is, is still quite small, and uses features ill-suited to distinguishing large-scale polities.

Three points raised by this analysis warrant further discussion. The first has to do with how social evolution has typically been explained. The second concerns the Old/New World divergence revealed here. Finally, we raise the possibility that this analysis points to new avenues for understanding social instability.

\section{Role of Population}

Archaeologists have long held different views on the importance of population growth in social evolution. Recently, for example, arguing from a perspective grounded in the small- and middle-range societies in the US Southwest, Kohler and Crabtree ${ }^{30}$ attempt to demonstrate that increasing social and political complexity is largely driven by population growth. From a point of view more connected with the ancient states of Mesoamerica, however, Feinman ${ }^{31}$ recognizes relatively strong correlations between population size and sociopolitical complexity, but terms the relationship "messy," arguing that it is significantly mediated by the "specific nature of the relational links between individuals" especially the specific organization of the institutions by which different societies are governed [31, p. 47]. Our findings here that growth in scale is dominant at the extrema of PC1 but that development of information processing and exchange mechanisms is critical in the middle range of PC1 - suggest that both of these perspectives can be accommodated.

Moreover, our analysis raises the possibility that the zone between the thresholds of Scale and Information is especially critical for the evolution and elaboration of institutions. Institutions are mechanisms "whose outcomes are rules of interactions" and they "provide a means for individuals to amalgamate 
dispersed information about resources and wants, and hence coordinate ... actions to reach an equilibrium that gives higher pay-offs" than would be possible in their absence [32, p. 3-4]. Institutions maintain and promulgate information about peoples' past behaviours - information that becomes more difficult to recover as group sizes grow [32, p. 4]. So institutions thrive on and deal in information, and will seek to improve the mechanisms for its storage and retrieval. Koji Mizoguchi describes Japan's Kansai region ca. A.D. 400 (Middle Kofun, PC1 score -0.57) as experiencing an increasing number of distinct fields of communication resulting in part from extension of political interests into neighboring regions of Korea coupled with attempts to institutionalize communications. By A.D. 500 (late Kofun, PC1 score 0.20) this region has crossed the Information Threshold into an "archaeology of bureaucracy" in which the elite were paving the way for "registration and direct control of commoners" [33, p. 308]. Within a century, by A.D. 600 (Asuka, PC1 score 1.64) the Kansai region hosted the rise of the palace, the introduction of Buddhism, the first national treasury, the introduction of Chinese-modeled fiscal policies and script, and formalization of the Ritsuryo law code.

\section{Diverging Old and New World Trajectories}

One unexpected finding is that few New World societies seem to cross the Scale Threshold, and therefore do not undergo as much pressure to develop information and exchange systems as societies that did. This suggests that if we want to understand the rarity of writing systems in the New World and the absence of coinage we first need to understand the factors limiting scale in many such societies. There are many candidates, including perhaps the absence in the New World of an inland sea that provided an efficient means of linking societies on its periphery. (But why could the Gulf of Mexico not have fulfilled that role?)

More promising as an explanatory factor is the general absence of animals in the Americas capable of carrying people or sizeable loads (also connected with the famous absence of the wheel as a practical device). It is notable that the only partial exception in the Seshat sample is the Cuzco NGA where camelids could carry loads, but not warriors. Not coincidentally we think, this is also the only New World society in this sample that crosses the Scale Threshold, and develops a recording system (the quipu) beyond mnemonic devices.

\section{Explaining Social Instability}

In most of the polities recorded in Seshat, there is a strong relationship between movement along the PC1 dimension, growth in scale, and growth of information-processing capacities — but not in all of them. Could some of the frequent collapses seen in societies be due to a polity's never developing sufficient information-processing capacities, so that it "gets stuck", entraining collapse through poor performance due to lack of external connectivity, internal coherence, or inability to compete with polities whose superior information-processing abilities have enabled more growth in size? While Seshat is currently too small to address whether such "correlational failures" should be taken seriously - since we can't assess the global degree of correlation convincingly - that may not be the case in the future.

Archaeologists have long noted the instability of societies of intermediate complexity. The Mississippian (late-prehistoric, maize-based) societies of the US Midwest and Southeast, traditionally characterized

as simple or complex chiefdoms, commonly cycled unstably between these two organizational forms ${ }^{34}$ or, in a related vision, oscillated between more dispersed and more concentrated power distributions in a near-constant churn of fission-fusion ${ }^{35}$. Proximate mechanisms driving this instability appear to have included both endogenous and exogenous factors: contention over chiefly succession, the fortunes of war, and climatically induced variability in the maize production fueling their tribute-based hierarchies. While such factors may in fact be sufficient, the results here suggest the further possibility that mismatch among 
aspects of social development should be considered as a generic cause of instability.

For example, Fried [36, p. 225] surmised that stratified societies lacking state institutions "must be one of the least stable models of organization that has ever existed" since he thought that stratified communities would have to quickly develop ever more powerful institutions of political control to maintain the differential access to basic resources that defines systems of stratification. Analyzing such problems as failures of correlation among the sociopolitical features of a polity might provide a more general view on the nature of collapses without clear exogenous triggers. Pursuing such a vision is a topic for future research.

\section{Methods}

Methods are described in appropriate sections of the Supplementary Information.

\section{References}

1. Richerson, P. J., Boyd, R. \& Bettinger, R. L. Was agriculture impossible during the Pleistocene but mandatory during the Holocene? A climate change hypothesis. Am. Antiq. 66, 387-411 (2001).

2. Bettinger, R. L. Macroevolutionary theory and archaeology: Is there a big picture? In Prentiss, A., Kuijt, I. \& Chatters, J. C. (eds.) Macroevolution in Human Prehistory, 275-295 (Springer New York, New York, NY, 2009).

3. Kavanagh, P. H. et al. Hindcasting global population densities reveals forces enabling the origin of agriculture. Nat. Hum. Behav. 2, 478-484 (2018).

4. Bocquet-Appel, J.-P. When the world's population took off: the springboard of the neolithic demographic transition. Science 333, 560-1 (2011).

5. Kohler, T. A. \& Reese, K. M. Long and spatially variable Neolithic Demographic Transition in the North American Southwest. Proc. Natl. Acad. Sci. 111, 10101-6 (2014).

6. Childe, V. G. The Urban Revolution. Town Plan. Rev. 21, 3-17 (1950).

7. Smith, M. E. V. Gordon Childe and the Urban Revolution: A historical perspective on a revolution in urban studies. Town Plan. Rev. 80, 3-29 (2009).

8. Morris, I. Why the West Rules-For Now: The Patterns of History, and What They Reveal about the Future (Profile Books, London, 2010).

9. Kohler, T. A. et al. Greater post-Neolithic wealth disparities in Eurasia than in North America and Mesoamerica. Nature 551, 619-622 (2017).

10. Borgerhoff Mulder, M. et al. Intergenerational wealth transmission and the dynamics of inequality in small-scale societies. Science 326, 682-8 (2009).

11. Mattison, S., Smith, E. A., Shenk, M. K. \& Cochrane, E. E. The evolution of inequality. Evol. Anthropol. 25, 184-199 (2016).

12. Sebastian, L. \& Lipe, W. D. Perspectives from the Advanced Seminar. In Sebastian, L. \& Lipe, W. D. (eds.) Archaeology \& Cultural Resource Management, 283-297 (School for Advanced Research Press, Santa Fe, NM, 2009).

13. Denaire, A. et al. The cultural project: Formal chronological modelling of the Early and Middle Neolithic sequence in Lower Alsace. J. Archaeol. Method Theory 24, 1072-1149, DOI: 10.1007/ s10816-016-9307-x (2017). 
14. d'Alpoim Guedes, J. A., Crabtree, S. A., Bocinsky, R. K. \& Kohler, T. A. Twenty-first century approaches to ancient problems: Climate and society. Proc. Natl. Acad. Sci. 113, 14483-14491, DOI: 10.1073/pnas.1616188113 (2016). http://www.pnas.org/content/113/51/14483.full.pdf.

15. Adams, R. M. The Evolution of Urban Society: Early Mesopotamia and Prehispanic Mexico (Aldine, Chicago, 1966).

16. Trigger, B. G. Understanding early civilizations: a comparative study (Cambridge University Press, Cambridge, UK, 2003).

17. François, P. et al. A macroscope for global history. seshat global history databank: a methodological overview. Digit. Humanit. Quarterly, http://hdl.handle.net/2299/1748 (2016).

18. Turchin, P. et al. Seshat: The global history databank. Cliodynamics 6, 77-107 (2015).

19. Turchin, P. Fitting dynamic regression models to Seshat data. Cliodynamics 9, 25-58 (2018).

20. Turchin, P. et al. Evolutionary pathways to statehood: Old theories and new data. SocArXiv Prepr. DOI: 10.31235/osf.io/h7tr6 (2018).

21. Whitehouse, H. et al. Complex societies precede moralizing gods throughout world history. Nature DOI: 10.1038/s41586-019-1043-4 (2019).

22. Turchin, P. et al. Quantitative historical analysis uncovers a single dimension of complexity that structures global variation in human social organization. Proc. Natl. Acad. Sci. 115, E144-E151, DOI: 10.1073/pnas.1708800115 (2018). http://www.pnas.org/content/115/2/E144.full.pdf.

23. Turchin, P. \& Korotayev, A. V. Population dynamics and internal warfare: A reconsideration. Soc. Evol. Hist. 5, 112-147 (2006).

24. Peregrine, P. N. Outline of Archaeological Traditions (HRAF Press, New Haven, Connecticut, 2001).

25. Peregrine, P. N. Toward a theory of recurrent social formations. In Sabloff, J. A. \& Sabloff, P. L. W. (eds.) The Emergence of Premodern States: New Perspectives on the Development of Complex Societies, 271-295 (SFI Press, Santa Fe, NM, 2018).

26. McGhee, G. R. The Geometry of Evolution: Adaptive Landscapes and Theoretical Morphospaces (Cambridge University Press, Cambridge, UK, 2006).

27. Diamond, J. The World Until Yesterday: What can we learn from traditional societies? (Penguin, New York, NY, 2012).

28. Richerson, P. \& Boyd, R. The evolution of human ultrasociality. In Eibl-Eibesfeldt, I. \& Salter, F. (eds.) Ethnic Conflict and Indoctrination: Altruism and Identity in Evolutionary Perspectives, 71-95 (Berghahn, Oxford, UK, 1998).

29. Turchin, P., Currie, T. E., Turner, E. A. L. \& Gavrilets, S. War, space, and the evolution of old world complex societies. Proc. Natl. Acad. Sci. 110, 16384-16389 (2013).

30. Kohler, T. A. \& Crabtree, S. A. Village and polity formation in pueblo societies: How population growth channels social processes in neolithic societies. In Sanders, L. (ed.) Peupler la Terre : De la Préhistoire à l'ère des métropoles, 173-192 (Presses Universitaires François Rabelais, Paris, France, 2017).

31. Feinman, G. M. The emergence of social complexity: Why more than population size matters. In Carballo, D. M. (ed.) Cooperation and Collective Action: Archaeological Perspectives, 35-56 (University Press of Colorado, Boulder, 2013). 
32. Powers, S., van Schaik, C. \& Lehmann, L. How institutions shaped the last major evolutionary transition to large-scale human societies. Phil. Trans. R. Soc. B 371, 20150098 (2016).

33. Mizoguchi, K. The Archaeology of Japan: From the Earliest Rice Farming Villages to the Rise of the State (Cambridge University Press, New York, NY, 2013).

34. Anderson, D. G. Fluctuations between simple and complex chiefdoms: Cycling in the late prehistoric Southeast. In Scarry, J. (ed.) Political Structure and Change in the Prehistoric Southeastern United States, The Ripley P. Bullen Series, Florida Museum of Natural History, 231-252 (University Press of Florida, Gainesville, FL, 1996).

35. Blitz, J. H. Mississippian chiefdoms and the fission-fusion process. Am. Antiq. 64, 577-592 (1999).

36. Fried, M. H. The Evolution of Political Society: An Essay in Political Anthropology (Random House, New York, NY, 1967).

\section{Acknowledgements}

This material is based upon work supported by the National Science Foundation under Grant No. SMA1620462 to DHW and TAK. We thank Laura Ellyson, Department of Anthropology, Washington State University, for advice on the structure and content of Seshat data, Peter Turchin and the Seshat project for providing the dataset used here, Henry Wright for general inspiration, and the Santa Fe Institute for support. TAK also acknowledges support and hospitality from the Research Institute for Humanity and Nature, Kyoto, while this was completed. 


\section{Supplementary Information for "From Scale to Information and Back: Human Social Evolution in the Holocene"}

Jaeweon Shin, Michael Holton Price, David H. Wolpert, Hajime Shimao, Brendan Tracey, and Timothy A. Kohler

\section{Contents}

1 Overview of Seshat $\quad 1$

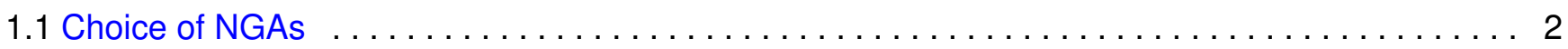

1.2 Choice of Variables and Byproducts of Imputation $\ldots \ldots \ldots \ldots \ldots \ldots \ldots \ldots \ldots \ldots \ldots$

2 Previous Seshat Research $\quad 3$

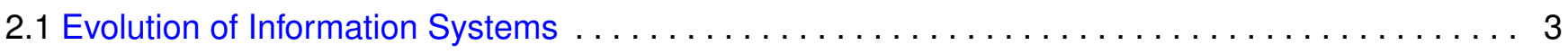

2.2 Evolution of Specialized Governance Structures $\ldots \ldots \ldots \ldots \ldots \ldots \ldots \ldots \ldots \ldots \ldots \ldots$

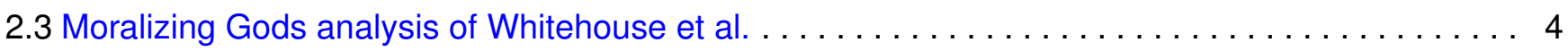

3 Bimodality $\quad \mathbf{5}$

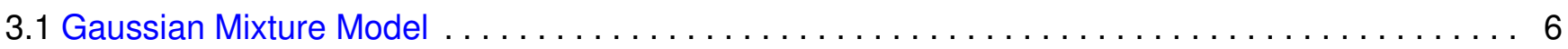

3.2 Discrete Markov transition model $\ldots \ldots \ldots \ldots \ldots \ldots \ldots \ldots \ldots \ldots \ldots \ldots \ldots \ldots \ldots \ldots$ Model Details

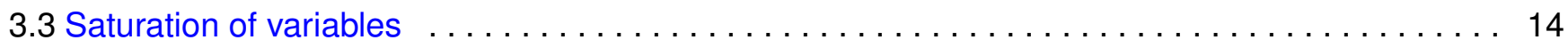

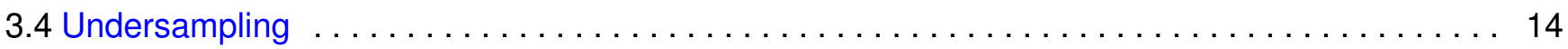

4 Plots of PC1 by Time $\quad 16$

$\begin{array}{lr}\text { References } & 19\end{array}$

\section{Overview of Seshat}

The Seshat project was initiated in 2009 to develop "a general resource for testing theories about sociocultural evolution and cliodynamics" [1, p. 59]. Here we provide an overview of the Seshat database including its structure (1.1) and variables (1.2), identifying some issues raised by the imputation procedure in ${ }^{2}$. Seshat is an evolving database, and so this summary largely refers to the version of Seshat used in ${ }^{2}$. In 2 we briefly summarize three more recent Seshat publications; 2.3 complements the section "Appearance of Moralizing Gods" in the main text. The remainder of this SI analyzes the source(s) of the bimodality in polity scores on PC1 (3) and for reference plots the PC1 scores through time for each NGA (4).

The Seshat database divides the world spatially into distinct "Natural Geographic Areas" (NGAs) and then temporally subdivides these by "polities" each of which lasts for one or more centuries. An NGA is a purely geographic unit, defined as an area roughly $100 \mathrm{~km}$ by $100 \mathrm{~km}$ "delimited by naturally occurring geographical features (for example, river basins, coastal plains, valleys, and islands)" [2, p. 6]. Examples of NGAs are the Cambodian Basin, the Konya Plain, and the Big Island of Hawaii. Each NGA is controlled by a sequence of polities, with at most one NGA controlling the polity at any time. A polity is "an independent political unit that ranges in scale from groups organized as independent local 
communities to territorially expansive, multi-ethnic empires" [2, p. 2]. ${ }^{1}$

Generally, polities are defined more granularly than in colloquial speech, and a new polity is assigned when there is a significant sociopolitical realignment. For example, Seshat recognizes eight polities in the Kingdom of France from Charlemagne to Louis XIV. Seshat encodes measurements of societal complexity (where available) at 100-year intervals for each NGA and its associated controlling polity. The relationship between NGAs, centuries, and polities can be clarified by the example of Iceland. Iceland has are four observations, one every century from AD 1000-1300 inclusive. The first three observations (AD 1000-1200) are coded as a single polity (the Commonwealth), and the last (AD 1300) as a separate polity (part of the Danish Kingdom).

\subsection{Choice of NGAs}

A subset of NGAs in Seshat was used in the analysis of Turchin and colleagues ${ }^{2}$. This set was chosen to maximize geographic extent and diversity in social organization. In particular, 30 NGAs were sampled from 10 world regions (e.g., South America; Africa). From each world region three NGAs were selected to provide one example each of a place where complexity appeared early, intermediate, and late in date. The resulting dataset contains 414 distinct observations across NGAs at 100-year intervals. Seshat is an evolving dataset with the versions resulting in publications available for download at http://seshatdatabank.info/datasets/.

\subsection{Choice of Variables and Byproducts of Imputation}

The Seshat database now contains over 1500 variables. However, only a subset was used for recent articles analyzing complexity (and by us). Turchin and colleagues ${ }^{2}$ chose 51 variables related to social complexity that they believe can be reliably coded even for archaeological contexts. These variables are imputed into a set of nine distinct summary variables, called "complexity characteristics" (CCs). The nine CCs measure: (1) polity population, (2) extent of polity territory, (3) population size of largest urban center, (4) hierarchical complexity (administrative, religious, military, settlement), (5) government, (6) infrastructure, (7) information systems, (8) specialized literature, and (9) monetary system (see also Table 1, main text).

A problem with this imputation is that there are frequently one or more of the original variables for which values are missing. This can be due to incomplete research, expert disagreement, or other uncertainties about the correct value. Missing values are especially common for earlier polities for which less information is available. Rather than assign a value and create one canonical imputation, instead a distribution of possible values is assigned. These distributions are then sampled 20 times, to produce 20 replicates of the dataset [2, p. 7]. The imputation is performed on each replicate, producing 20 different sets of CC values. These replicates are used to produce confidence intervals on the proportion of variance explained by each PC, the component loadings, and the values of the PCs for each polity. The authors graciously provided the 20 imputed datasets, forming the basis of our analysis here. The complete dataset contains 8280 rows (414 observations $* 20$ imputations), and 13 columns. The columns are: the name of the NGA, an identifier for the polity, the century of the observation, the value for each of the nine CCs (the result of imputation), and the imputation replicate identifier.

It is visually apparent that there are artifacts in the data shown in Supplementary Figure 2. The data appear to exhibit noticeable "streaks" at approximately a thirty-degree angle from the vertical. We examined the cause of these streaks to ensure they are not a significant contributor to our findings. We first examined if the streaks are caused by strong correlations among a (set of) CCs. We examined this by

\footnotetext{
${ }^{1}$ Seshat uses a representative quasi-polity when more than one political entity existed in an NGA at one time, or when control of the polity switched rapidly among political entities $-\operatorname{see}^{1}$ for further details. Following Seshat usage ${ }^{2,3}$, we do not distinguish quasi-polities from polities in our analysis.
} 
reproducing Supplementary Figure 2 but zeroing out the contribution from all combinations of 1, 2, and 3 CCs. The character of the streaks (primarily the orientation) changes when certain CC combinations are removed, but all subsets still contain visible streaks, which implies the streaks are not caused by strong CC combinations.

This also implies they are not caused by the fact that some CCs are more "discrete" than others, for instance measured in integer increments from 1 to 5 rather than a more continuous measure. Instead, these streak artifacts are caused by the procedure used by ${ }^{2}$ to generate the 20 dataset replicates. Each streak is, in fact, a single polity with its 20 replicates. The apparent directionality of the streaks arises from the uneven uncertainty assigned to each CC. Population measures, for instance, are specified in a range that is sampled, while Money Level CC was not assigned an uncertainty. In between are multiple uncertainties that are averaged into a single $\mathrm{CC}$, and so their combined variance is reduced compared with population. These streaks give a visual representation of the effective uncertainty assigned to each polity. We find that the measured uncertainty in the Seshat data does not impact the results of the analysis in this work, nor the analysis of Turchin and colleagues ${ }^{2}$. The variation in each polity is small compared with the variation between polities. This level of measured uncertainty does not affect the existence of two strong clusters, and this noise, if anything, reduces the strength of the primary principal component rather than enhancing it. Nevertheless, we incorporate the uncertainty from the different imputations in reporting the rest of our results.

\section{Previous Seshat Research}

We do not attempt a comprehensive review of the rapidly growing set of articles from this project. Instead, we summarize four recent, relevant articles. The foundational article in this line of inquiry ${ }^{2}$ describes and analyzes the dataset we consider here. The major finding is the primary principal component (PC) captures $77 \%$ of the variation in the "complexity components" (CCs), with approximately equal loading by all nine CCs onto that first PC. Small-scale polities (typically earlier in time) have generally low scores when projected onto this primary PC, while larger-scale polities (typically later in time) generally have high projection scores. The location of any single polity along this one, nine-dimensional PC might plausibly serve as a useful scalar measure of how complex, or possibly "developed", that polity was, in the sense of sharing features with large-scale rather than small-scale societies.

\subsection{Evolution of Information Systems}

This time-stamping is used profitably in two other recent papers from the Seshat group. The first ${ }^{4}$ uses multiple linear regression to explain variation through time in the information complexity CC (Info). Potential independent variables lead the dependent by one century so that causal inferences respect time's arrow. There is also an autoregressive term for the dependent, to examine the extent to which earlier values for Info predict the current value. Finally, there are terms examining correlations due to shared culture history (proxied as language family) and spatial diffusion. The best linear model (that with the smallest AIC) identified first- and second-order lags of Info to be the most important predictors of Info, followed by phylogeny, Money, and PolPop (polity population), all with positive slopes. Money is a scale reflecting the "most sophisticated" monetary instrument present in the coded polity (0: none, 1: Articles, 2: Tokens, 3: Precious metals, 4: Foreign coins, 5: Indigenous coins, 6: Paper currency) [4, p. 39]. Broadly similar results were achieved when quadratic (squared) terms for the regressors were introduced although the Lag1 squared term for the autoregressive version of the independent variable bore a negative sign, possibly suggesting a "regulatory" effect for this term: "when Info gets too high, this term "steps on the brakes" to impede its further increase" [4, p. 51]. 


\subsection{Evolution of Specialized Governance Structures}

Another recent paper ${ }^{5}$ employs the same approach to analyze the evolution of the internal specialization of governance structures (Gov) which sums 11 binary variables reflecting the presence/absence of various specific offices, government buildings, examination systems, etc. [5, p. 3]. Here, the lag1 version of Gov is by far the most important variable in the nonlinear regression, followed by the phylogeny proxy, a quadratic version of Gov, and (log-transformed) polity population (all signs positive except for Gov). The only other independent variable with a significant $t$ value is Time, the absolute date of each time step. The authors interpret the inclusion of this variable in all the best-fitting models to indicate that important unobserved variables remain to be discovered and added to the model.

As valuable as these two analyses are, particularly since they take time explicitly into account, they deal with just one (dependent) variable at a time. Our re-analysis of the data in the main paper $^{2}$ where all the variables are considered simultaneously, is designed to see what gains can be made when that analysis is performed in a dynamic mode.

\subsection{Moralizing Gods analysis of Whitehouse et al.}

In the main text we summarize an analysis by Whitehouse and colleagues ${ }^{3}$ which uses the Seshat dataset to test the hypothesis that social complexity increases more rapidly following the appearance of moralizing gods. Here we provide more detail on how they coded their Belief in Moralizing God (BMG) variable which they added to the dataset we discussed above for this analysis. (The coding for this variable has been criticized by ${ }^{6}$ for different reasons.) For each time-stamped observation in each NGA sequence, there are three possible codes for Moralising Gods: NA / Missing, 0 / Absent, and 1 / Present. Missing is common for early dates (and, in general, lower PC1 values) since it is typically difficult to determine the presence of Moralizing Gods based on archaeological data unsupplemented by textual evidence.

The 30 sequences (i.e., the 30 NGAs througn time) analyzed by ${ }^{3}$ can be classified into the following 7 groups based on whether Moralising Gods are ever present and, if so, the nature of the first point in each sequence for which Moralising Gods are present:

- "Missing to Present", i.e., the Moralising Gods data is missing for early times for the NGA in question, but is filled in for later times, with the value 'Present' [12]: Big Island Hawaii, Deccan, Kachi Plain, Kansai, Konya Plain, Latium, Niger Inland Delta, Orkhon Valley, Paris Basin, Sogdiana, Susiana, Upper Egypt

- "Always Missing" [4]: Cahokia, Finger Lakes, Southern China Hills, Valley of Oaxaca

- "Always Present" [7]: Cambodian Basin, Central Java, Chuuk Islands, Ghanaian Coast, Iceland, Kapuasi Basin, Yemeni Coastal Plain

- "Missing to Absent", i.e., the Moralising Gods data is missing for early times for the NGA in question, but is filled in for later times, with the value 'Absent' [2]: Cuzco, North Colombia

- "Always Absent" [4]: Garo Hills, Len River Valley, Lowland Andes, Oro PNG

- "Absent to Present" [1]: Middle Yellow River Valley

As mentioned in the main text, in a pre-processing step Whitehouse and colleagues ${ }^{3}$ discarded all NGAs that did not have data points both before and after the appearance of Moralising Gods. This introduces a strong statistical bias in favor of NGAs where Moralizing Gods arise late (and so are more likely to arise after the first data points in Seshat for that NGA). Indeed, the exclusion of NGAs with the 
pattern, "Always Present" excludes a number of NGAs for which Moralizing Gods were Present with early calendar dates and, often, with low PC1 values. In fact, the only NGAs that survived their pre-processing step were the Middle Yellow River Valley NGA (the only NGA that exhibits the pattern, "Absent to Present") and 11 of the 12 NGAs that have the pattern, "Missing to Present". 2

\section{Bimodality}

Our reanalysis of the Seshat dataset reported by Turchin ${ }^{2}$ was originally motivated by the apparent bimodality in the frequency of polities projected onto their PC1 as illustrated in Supplementary Figure 1. This was first of all a concern: the primary formal justification for an analysis based on PCA (as in ${ }^{2}$ ) is the assumption that the data are generated by IID sampling a single Gaussian. The presence in these data of two highly distinct clusters is therefore problematic. Indeed, as shown in Supplementary Figure 1, the clusters in PC1 appear very similar, being accurately described by a mixture of two Gaussians of very similar widths, distinguished only by their mean values on PC1. Other results emerging from the PCA, such as the finding that PC1 captures " $77 \%$ of the variability" become unreliable given this data structure. That is one justification for our decision to pay more attention to PC2, since, likewise, it may be more important than the statistics generated in ${ }^{2}$ suggest. Accordingly, as a conservative first step, in this paper we consider the Seshat dataset projected onto the top two PCs.

The second reason the bimodality is of interest however is that - if not an artifact of sampling or analysis - it may suggest a fundamental division of societies, perhaps into "simple" and "complex" groups, that additionally might be regarded as fundamental basins of attraction for the dynamics of social groups as they move through the Holocene. (For example, on the basis of a somewhat similar analysis Peter Peregrine ${ }^{7}$ has recently argued for the existence of four modes in sociopolitical organization.) One of these clusters (with a modal score around -4 on PC1) corresponds to polities of modest demographic scale, often but not invariably early in time. The other cluster (with a modal score around 2 on PC1) corresponds to polities of larger demographic scale, generally appearing later in time. As a shorthand we will refer to these as the "early" and "late" clusters, though this is not invariably true in calendar years. The only polities that reach the scale required for stable membership in the late cluster, at least in this sample, are from the Old World (Eurasia, Africa, and Oceania).

Turchin and colleagues ${ }^{2}$ also recognized these two clusters. On pp. 26-28 of the supplemental information they note "some initial support for the idea" of clusters in the distribution of societies along PC1 "with a relatively large distance between two main clusters". Figure SI11 in that paper suggests that two modes begin to develop along the PC1 dimension by $1000 \mathrm{BC}$ and are quite prominent by AD 1 . Similarly, Turchin et al. [5, p. 13] report that "there may be two clusters in the governance-population phase space, where trajectories tend to spend most time. The transition between the two clusters, on the other hand, happens quickly."

We now present several analyses that suggest that the bimodal distribution of points along PC1 arises is likely not of substantive interest, though this structure still raises issues for the strict interpretation of the proportions of variance explained by the various PCs. The bimodality is not the result of the streak artifacts discussed in 1.2. We also show that the bimodality is not the result of saturation of the variables recorded in the Seshat dataset at their extremal values as societies develop. (Such saturation may however contribute to the accumulation of datapoints in the right-hand cluster). There were also some possibilities we could not reject. In particular, we could not conclude that there is not an attractor of the underlying dynamics (i.e., a "sink") in the right-hand cluster.

\footnotetext{
${ }^{2}$ Big Island Hawaii was excluded because it has Present for the final observation of its sequence ca. A.D. 1800, which was attributed to colonial influence.
} 


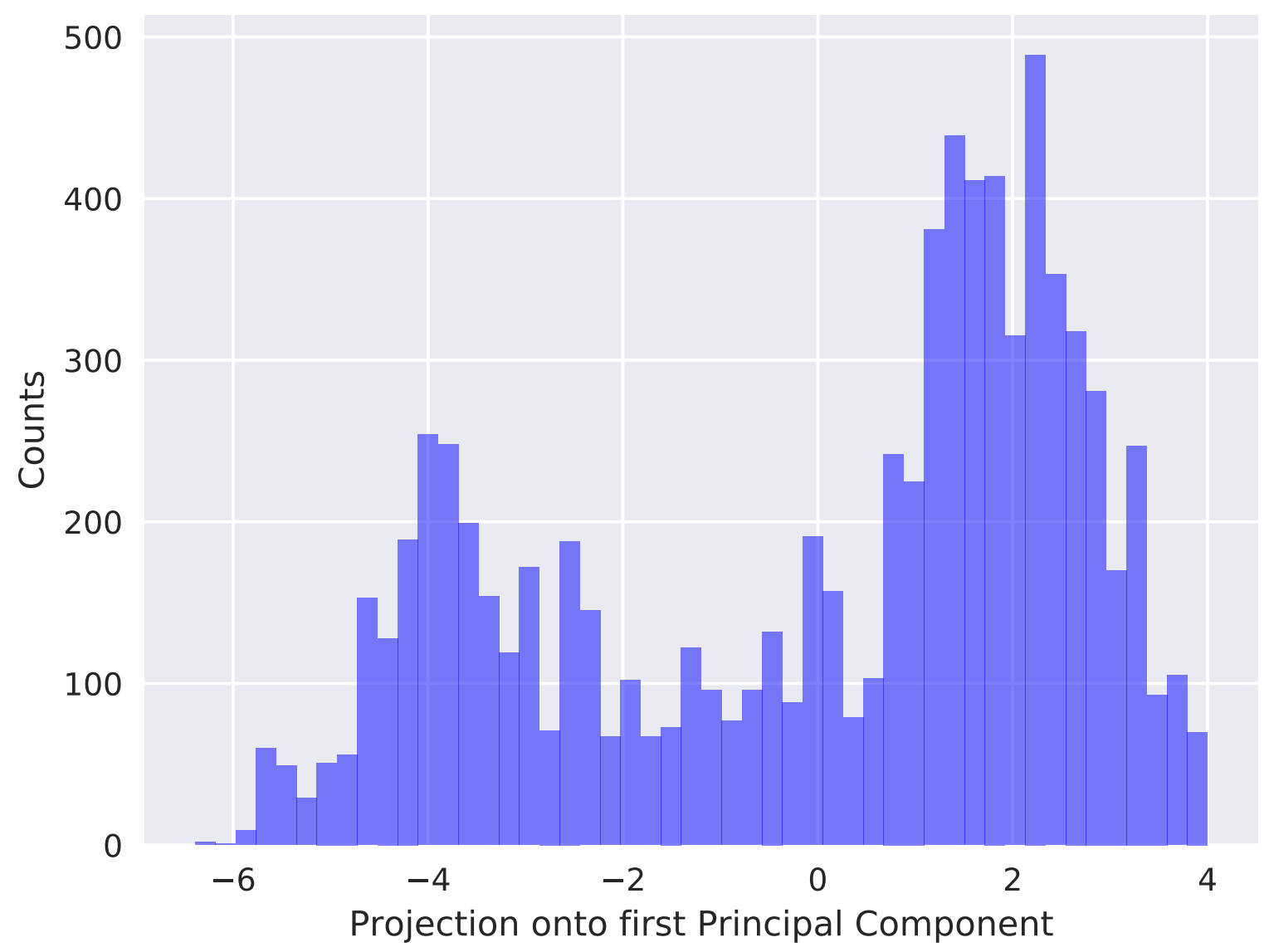

Supplementary Figure 1. Histogram of polities projected onto PC1. Each imputation comprises a separate point in the underlying data for the histogram.

\subsection{Gaussian Mixture Model}

To investigate the bimodality in more detail we began by fitting a two-component Gaussian mixture model to the full 9-dimensional (9D) imputed Seshat dataset. Supplementary Figure 2 presents a 2D scatterplot of PC1-PC2, with the coloring of the points determined by which 9D Gaussian cluster each point most likely belongs to. We define cluster membership for each point as its maximum likelihood cluster, i.e., the Gaussian with the higher membership probability. (Note that the true data are 9-dimensional, and the full data are used to define membership, given the seemingly non-monotonic clustering classification.)

Moreover, as shown in Supplementary Figure 3, most of the data points are included in the $<99 \%$ threshold, meaning that most points exclusively belong to one of the two clusters according to our Gaussian Mixture Model. This result suggests that the two clusters we observed in the data are clearly distinguishable.

To check that the two Gaussians were not caused by noise, we ran bootstrapping on the following values: 1) the percentage of the largest eigenvalue in the first and the second Gaussians, 2) angle between the two main eigenvectors of the two Gaussians, corresponding to the largest eigenvalue in each Gaussian, and 3) angle between Turchin's main principal axis and the main eigenvector of the first Gaussian and angle between Turchin's main principal axis and the main eigenvector of the second Gaussian. Note that 


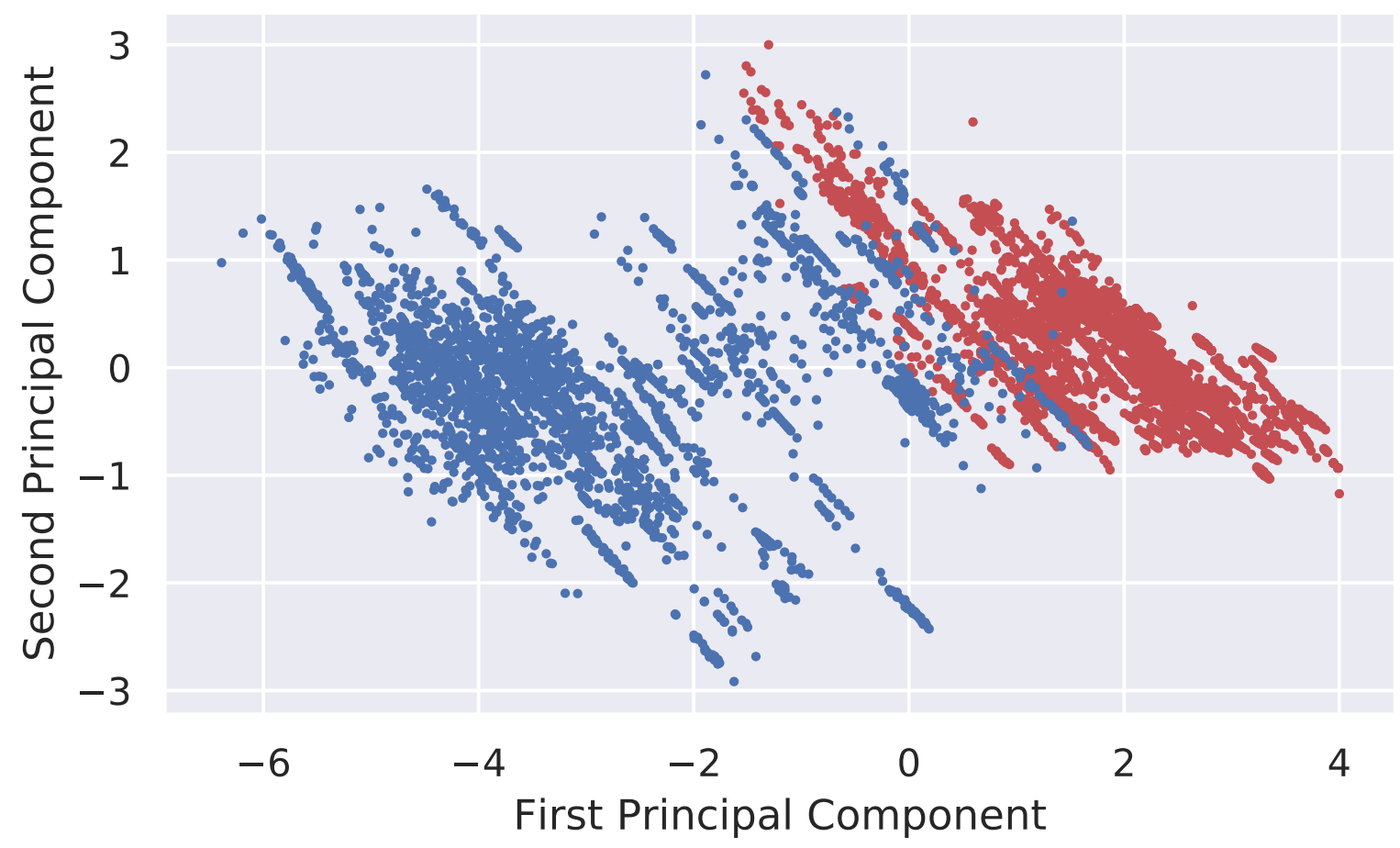

Supplementary Figure 2. Scatterplot of polities projected onto the first and second PC. The left (blue) and right (red) clusters are generated by fitting a two-component Gaussian mixture to the 9-dimensional PC data and determining, based on the mixture densities, whether a point belongs to the first or second cluster. The overlap between points in the cluster occurs because the 9-dimensional fit is displayed projected into the 2-dimensional PC1-PC2 space. In fact, a point belongs exclusively to one of the two clusters as suggested by Supplementary Figure 3. Note the pronounced "streaking" from top left to bottom right which appears to be due to the imputation procedure.

all these values were obtained by fitting two Gaussians in the 9-dimensional imputed Seshat data matrix.

The methods for calculating the above listed properties of the Gaussians are as follows. 1) To compute the percentage of the largest eigenvalue in the two Gaussians, we first calculated 9 eigenvalues for the covariance matrix of each of the two fitted Gaussians. For each Gaussian, we then selected the eigenvalue with highest value and divided it by the sum of all 9 eigenvalues to obtain the percentage of the largest eigenvalue. 2) To compute the angle between the two main eigenvectors, we selected the eigenvector that corresponded to the largest eigenvalue in each of the two Gaussians. Then, we calculated the angle between the two vectors using the cosine formula. 3) Computing the angle between Turchin's main principal axis and the main eigenvectors of the first and the second Gaussian follows exactly the same procedure as above, by taking the main principal axis vector and computing the angle between that vector with the main eigenvectors of the two Gaussians. 

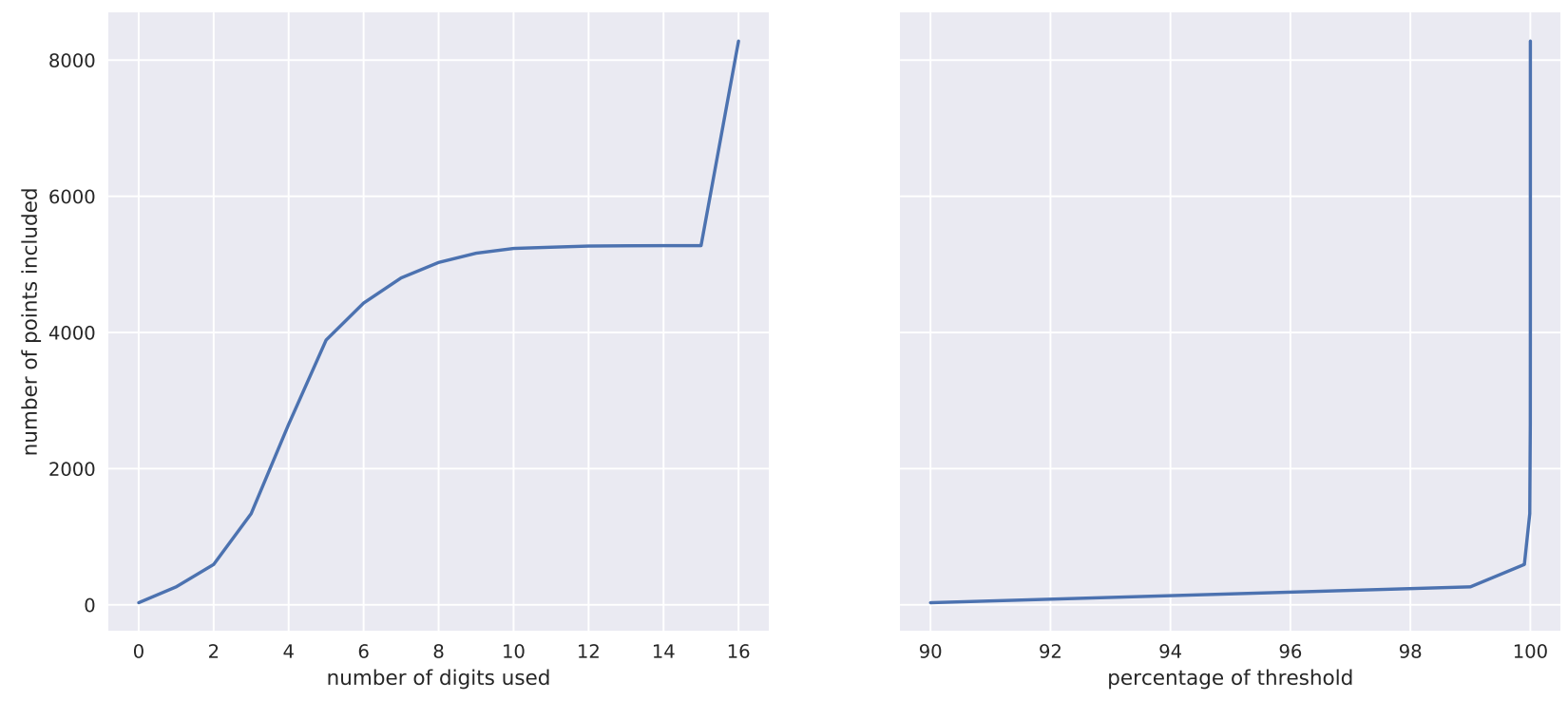

Supplementary Figure 3. Result for verifying the robustness of the two Gaussian clusters. After fitting the Gaussian mixture model with two components, using the predicted posterior probability for each component for each data point, we determine how many points have above the given threshold probability of belonging exclusively to one of the two components. Left: total number of points included in either of the clusters based on the number of digits used for the threshold probability (e.g., 6 digits for the threshold means that the threshold was 0.999999). Note that in this left-hand plot, when the number of digits used for the threshold reached 16, every data point was included in the clusters due to numerical overflow error. Right: number of points included in the two clusters when changing the threshold of the probability value (quantified as a percentage) for belonging exclusively to one or the other of the two clusters (e.g., "90 percent threshold" picks out data points that have less than $90 \%$ probability of belonging exclusively to either of the two clusters).

The procedure for bootstrapping was as follows. First, for each of the methods listed, we randomly resampled the original 9-dimensional imputed data matrix and obtained 5000 matrices that are of the same dimension as the original matrix. Then, for each of these 5000 matrices, we computed the statistic of our interest (e.g., angle between the two main eigenvectors) and obtained an array of resulting values for the statistic. We plotted the values calculated from resampled matrices, as well as plotting a vertical line that indicates the value calculated from the original matrix. The result (Supplementary Figure 4) suggests that the outcome is robust, implying that the two Gaussians were not caused by noise.

So we wish to ask whether this the bimodality is of substantive interest, or some kind of an artifact of the statistical analysis or the data-generation methods. (Note that some of those data-gathering methods are implicit, arising in how archaeologists choose where to excavate and what variables to pay attention to. Others are explicit, for example the decision by the creators of Seshat to exclude many polities from the database to try to get uniform coverage over NGAs, or of the use of imputations to "fill in" holes in that database.) We examined several candidate explanations for the bimodality and were able to reject several but not all of them.

\subsection{Discrete Markov transition model}

Underneath these statistical issues lurks a deeper point. Imagine for a moment that the time-stamping of all the polities were randomly re-assigned while keeping all the other attributes for each polity intact. 

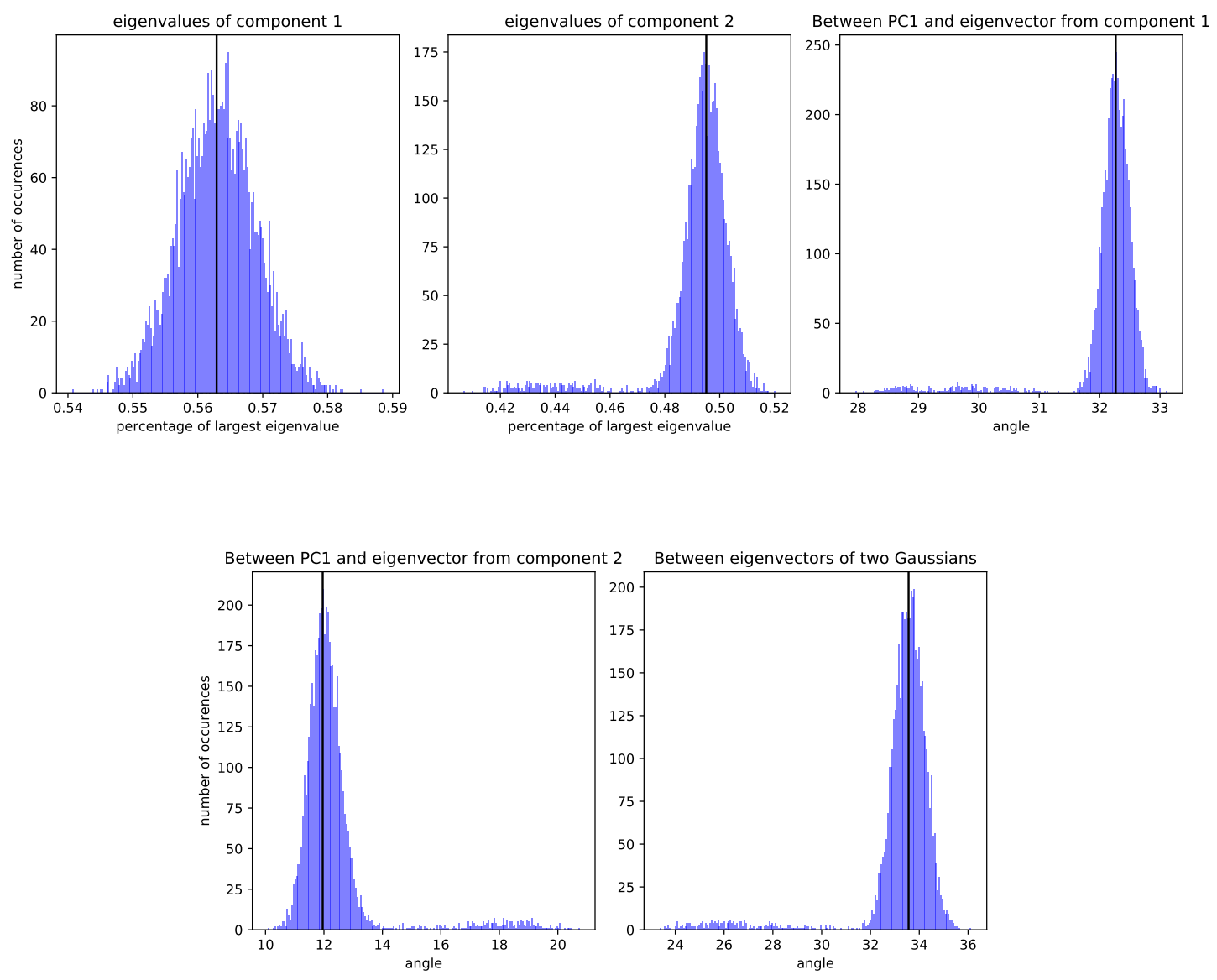

Supplementary Figure 4. Bootstrapping results for various statistics to verify the robustness of the two Gaussian clusters. (Top Left) The percentage of largest eigenvalue with respect to the sum of all eigenvalues in the first Principal Component. (Top Middle) The percentage of largest eigenvalue with respect to the sum of all eigenvalues in the second Principal Component. (Top Right) The angle between the first Principal Component and the eigenvector of the first Principal Component corresponding to the largest eigenvalue. (Bottom Left) The angle between the second Principal Component and the eigenvector corresponding to the largest eigenvalue in the second Principal Component. (Bottom Right) The angle between the two eigenvectors from the first and the second Principal Component, each corresponding to the largest eigenvalues with respect to each component.

A PCA would still "explain" $77 \%$ of the (static) patterning in the data, but each polity's movement along the continuum defined by PC1, over time, would be random. In that case, would we think that PC1 provides a useful summary of sociopolitical development? Probably not, since we understand (sociopolitical) evolution as an historical process. What really counts for understanding such a process is not the proportion of variability explained in a static pattern. Rather the proportion of the variability in the dynamics is what we ought to be interested in. Indeed, as described below, many aspects of static patterns can be misleading, leading to suppositions about the dynamics from the pattern that in fact are contravened 
if one directly analyzes the dynamics. This observation alone warrants examination of the dynamics of the data, ideally in more dimensions than just PC1. This issue is not assessed by ${ }^{2}$ (except for noting after having performed the PCA that early polities tend to have lower values of PC1 than later polities).

To more directly address whether the dataset reflects variation in the underlying dynamics as one moves across PC1, we constructed a novel null-hypothesis technique. This technique is based on the observation that even if the underlying dynamics through PC space is a time-homogeneous Markov chain, the associated dwell times in the various parts of PC space could vary significantly. (After all, a single Markov kernel will give different dynamics of PC2 conditioned on PC1, depending on what that PC1 is.) That heterogeneity in dwell times might in turn cause the observed bimodality, due to the fact that datapoints are sampled uniformly in time. In essence, the uniformity of sampling in time would introduce an artifact of non-uniformity of the distribution over space. Similarly, a time-homogeneous Markov chain could be consistent with the hinge points in expected PC2 plotted vs. PC1, discussed in the main text.

In light of these considerations, we considered a null hypothesis that the bimodality is just the result of iterating a time-homogeneous Markov chain. To do this, first we bin PC1. We then estimate the conditional distribution of moving from one bin to another over a given (fixed) time interval, $\Delta t$. We then iteratively apply that conditional distribution to every datapoint in Seshat, exactly $t / \Delta t$ times (where $t$ is the number of years ago of the timestamp of that datapoint). We then compared the resultant distribution of points to the full Seshat dataset.

In other words, we considered the null hypothesis that the distribution of points in PC1 arises from a combination of the following phenomena:

a) Random variation across the NGAs in the PC1 value of the earliest polity within that NGA;

b) Random variation across the NGAs in the chronological time of that first PC1 value;

c) A general drift of polities from low to high PC1 values driven by a time-homogeneous Markov chain.

Our tentative conclusion is that the data are consistent with our null hypothesis, i.e., that these three phenomena by themselves can explain the bimodality in PC1. Moreover, the conditional distribution of next bin given current bin under the estimated Markov chain of item (c) is irreducible (by inspection see below). So there is a unique fixed point of this null hypothesis dynamics, i.e., there is no evidence for there being more than one "basin of attraction" of the dynamics.

In addition we performed a very preliminary analysis of the relationship between the precise details of a stochastic process traversing a space and the resultant "static pattern" given by sampling that process at regular time points (as was done in generating Seshat, where the sampling time points were one century apart). Obviously, if the expected speed of movement across PC1 is slow for a broad interval of PC1, then is fast in a following interval, and then slow again in a following, third broad interval, there would be an increased number of datapoints in that first broad interval as well as the third one, with few points in between (given that the datapoints are formed by sampling at times all one century apart). This would result in the kind of bimodality in PC1 we found in the Seshat dataset. Conversely, if mean speed across PC1 were uniform across PC1, and all other aspects of the dynamics were also uniform, there would be no such induced bimodality in the samples of PC1 values.

However, we realized that even if the mean speed is uniform across PC1, variation in the breadth of possible speeds from one point in PC1 to another can result in bimodality of the sort that characterizes the Seshat dataset. To see why, first note that the amount of time a given polity will spend in a given interval of PC1 as it moves across PC1 will depend on the inverse of the velocity in that region. Moreover, if we 
have two distributions over speed values that are both Gaussian with the same mean, but one of those distributions has a bigger variance than the other, the associated expected inverse speed will be greater in the distribution with a larger variance of speeds. This means that there will be a greater density of points sampled from a region of the space with a high variance of speeds than from a region of the space with a low variance of speeds. ${ }^{3}$

Accordingly, as a variant of our null-hypothesis test, we modified how we estimate the conditional distribution, to force no such variation across PC1 in the variance of the velocity from one point in PC1 to another. More precisely, we first estimated the average over all the bins of the variance of the speeds leaving that bin, $\sigma^{2}$. Then for each column probability vector $p$ in the conditional distribution matrix of next bin given current bin, we solved for the distribution $q$ with minimal Kullback-Leibler divergence to $p$ which has its variance of speeds equal to the global average of such variances, $\sigma^{2}$. We then replaced $p$ by $q$. Doing this for all columns in the original conditional distribution matrix gives a new conditional distribution matrix, which we could then apply to the starting polity position / times of the datapoints in Seshat, just as before. This variant of the null hypothesis appears to give a slightly worse fit to the actual full Seshat data than the original null hypothesis, in which the variances of speeds are allowed to vary across PC1. This leads us to conclude that there is weak but inconclusive evidence that variation in the variance of the speeds of movement across PC1 contributes to the bimodality.

While the static bimodal pattern can be (mostly) explained this way, the ultimate question for us is what patterns there are in the dynamics across the space. To a degree, this can be assessed by examining the Markov transition matrices made under our null models. However, given the limited amount of data in Seshat, it is a subtle exercise to construct such a model for more dimensions than just PC1. As an alternative, we can more directly investigate those patterns in the multi-dimensional dynamics, without binning the space, estimating conditional distributions, etc. We describe such a direct investigation of the dynamics in next two subsections.

\subsubsection{Model Details}

In light of the extremely small amount of data, we considered only the dynamics over PC1, and discretized those PC1 values into 10 bins. We then formalized our assumptions (a)-(c) in 3.2 with the following model:

1. NGAs are "born" (i.e., first possess polities of sufficient levels of social development to warrant inclusion in the Seshat database) by IID sampling a distribution $p_{0}$ over those 10 bins;

2. The chronological time of the birth of each NGA is given by IID sampling a distribution $\Delta\left(t_{0}\right)$;

3. All NGAs evolve across the bins over time-jumps of 500 years by sampling the same (timehomogeneous) conditional distribution $\pi$;

4. All data points, over all times, are subject to observational noise (including random removal from the dataset entirely) to generate the final dataset.

For simplicity, we estimated $\pi$ by applying a frequency-count estimator to the 500-year bin-to-bin transitions in Seshat (shown in Supplementary Figure 5). However, to be maximally conservative, we avoided estimating $\Delta($.$) and p_{0}($.$) , by pursuing the following procedure instead: First, for each NGA$ $n$, we found the earliest bin at which it is present in Seshat, $i(n)$. Let $T(n)$ be the number of 500-year

\footnotetext{
${ }^{3}$ As a simple illustration, if the speed of movement across a bin of width 1 is 1 , with probability 1 , then it will take 1 unit of time to traverse that bin. If instead the speed when traversing that bin is either 1.5 or .5 with equal probability, the mean speed is again 1 , but it will take on average $(2+2 / 3) / 2=4 / 3$ units of time to traverse the bin.
} 
blocks into the past of that earliest data point $i(n)$. We applied $\pi$ to $i(n)$ a total of $T(n)$ times, to generate a set of $T(n)$ bin values; doing this many times gave us a scatterplot of the PC1 values that we would expect to see for that NGA, if indeed its dynamics was given by $\pi$. Running this procedure for all of the NGAs gives an overall scatterplot which, if our model is correct, should look similar to the actual Seshat scatterplot. Supplementary Figure 6(a) shows the results from this sampling procedure, which clearly exhibits bimodality similar to the original data.

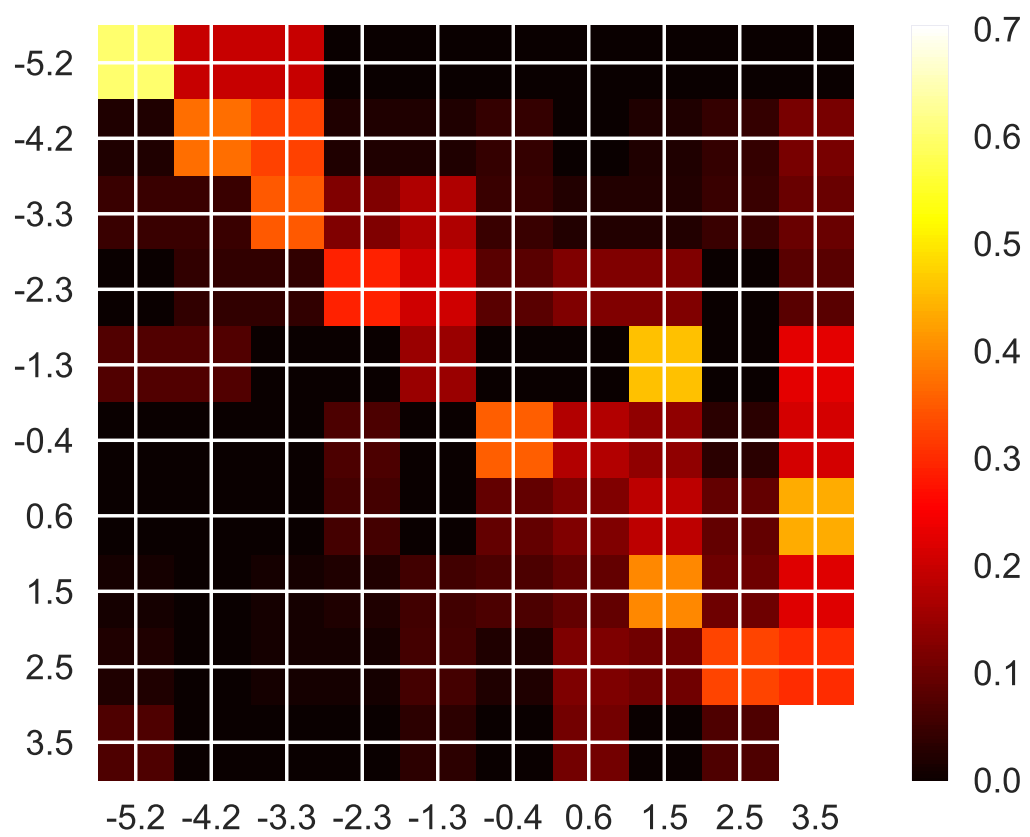

Supplementary Figure 5. The estimated transition probability $\pi$.

We used this simple model to investigate the dynamic cause of the bimodality. Recall that the Seshat datapoints were recorded for regularly sampled times one century apart. This means that the two clusters would arise if movement through PC1-PC2 space was smaller where the first and second clusters lie, and faster in between them. In other words, it would arise if there were heterogeneity in the mean velocities across PC1-PC2 space. In terms of our discussion just above, this would correspond to variation, across the rows of the transition matrix $\pi$, of the difference between the average column index specified by the distribution of that row and the row index itself, i.e., variation with bin $n$ of the difference $\left[\sum_{m} \pi(m \mid n) m\right]-n$.

Perhaps more surprisingly, even if the mean velocities do not vary, if the variance in the mean velocities varies in the appropriate way, then clusters would arise in regions where that variance is relatively large. To see this, note that the amount of time an NGA stays in a given PC1 interval is proportional to its inverse speed across PC1 in that interval. Therefore a Gaussian distribution in speeds will result in a non-Gaussian distribution in the amount of time an NGA stays in a given PC1 interval, i.e., a non-Gaussian distribution in the number of Seshat data points in that interval.

To test these possibilities, we first generated a new transition matrix that resembles the original matrix $\pi$, except the mean and/or variance of speeds across the bins was forced to be the same, no matter what 


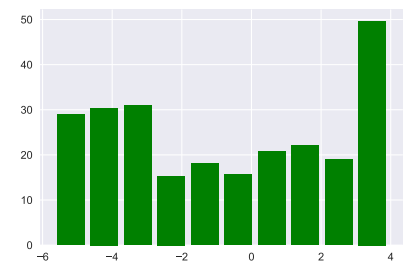

(a) Original data

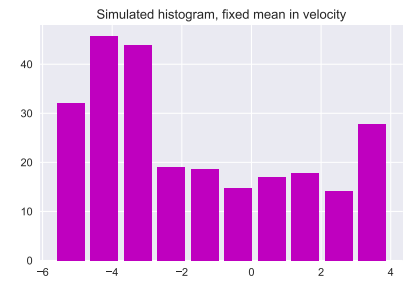

(b) Fixed-speed mean

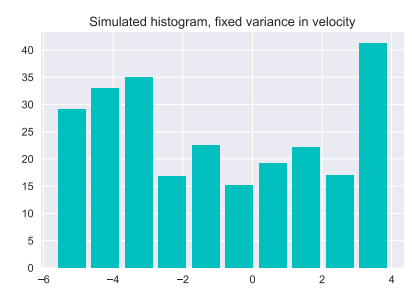

(c) Fixed-speed variance

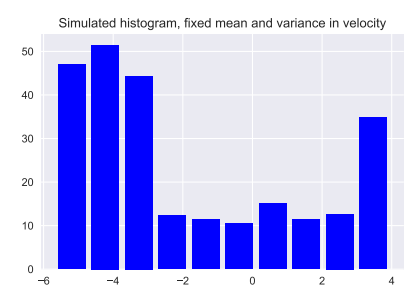

(d) Fixed-speed mean and variance

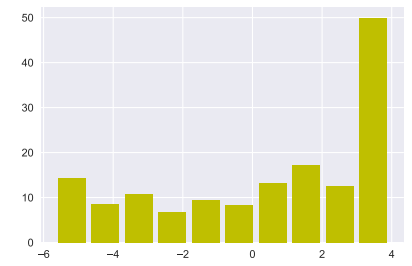

(e) From uniform distribution

Supplementary Figure 6. Histograms of (a) the original data and (b-e) simulated results from the Markov transition model. 
the originating bin. For each bin $n$, we generated the associated conditional distribution (i.e., row) of this alternative transition matrix, $\pi^{\prime}(. \mid n)$, by minimizing the KL-divergence between $\pi^{\prime}(. \mid n)$ and $\pi(. \mid n)$, subject to the constraints that the mean and/or variance of that new conditional distribution equals the associated value for the entire population, running over all $n$. Supplementary Figures 6(b), 6(c), and 6(d) simulate the histogram with constrained mean velocity, constrained variance in velocity, and both, respectively. The bimodality observed in the data (Supplementary Figure 6(a)) is still present in all these graphs. This suggests that variation in the mean and/or variance of speed in different PC1 regions cannot be the sole explanation for the bimodality, though we do not exclude the possibility that such variations contribute.

In addition to these possible explanations of the bimodality grounded in dynamics through PC1-PC2 space, it is also possible that the bimodality is simply caused by the sampling process that generated the initial distribution. That is, in Seshat the initial observation of NGAs is biased towards low PC1 values, which by itself would create the early cluster, even without any superimposed dynamic process. To see this, we simulated the histogram by setting $p_{0}$ to be a uniform distribution i.e. equal probability across bins, while keeping the transition matrix $\pi$ the same as estimated. In Supplementary Figure 6(e), we see that the size of the left cluster is reduced significantly from what is actually found in the Seshat data. This implies that non-uniformity of the initial values of PC1 for each NGA explains the left cluster, at least in part.

\subsection{Saturation of variables}

There are several ways in which the observed bimodality could result from a statistical artifact in the sampling or definition of the data or variables. One scenario is that the two peaks are generated from saturation of the variables (the nine CCs). If the saturation of different variables occurs at different rates, then peaks would be observed around the time when each variable reaches its upper limit and the growth of PC is temporarily slowed.

To test for this, we tried some simple simulations where the PC is a sum of two variables, both of which follow logistic growth $\left(L_{i} /\left(1 .+\exp \left(-k_{i} *\left(x-x_{i}^{0}\right)\right)\right), i=1,2\right)$ but with different parameters. Supplementary Figure 7 (a)-(c) shows how the two variables with different saturation times sum up. Assuming polities that follow this growth path are sampled uniformly, the resulting histogram is shown in Supplementary Figure 7 (d). While the two peaks can be generated from this logic, the right peak is at the right-most time period, in contrast to the data graphed in Supplementary Figure 1. It is also worth noting that it requires us to fine-tune parameters to create such peaks. Supplementary Figure 8 shows different combinations of saturation parameters. In general, the uniform sampling results in a plateau at the right rather than two spikes if both of the variables are saturated within the observed period.

\subsection{Undersampling}

Another possible explanation for the bimodality is selection bias in the data. For example, perhaps the polities of intermediate complexity were undersampled for some reason, such as lack of archaeological excavation or reporting for these polities, which might yield a bimodal distribution. To check that the bimodality (especially the peaks) were not caused by undersampling issues, we ran the following tests. Although it may be far-fetched to claim that these tests were sufficient to confirm that undersampling was not a problem, bimodality survived after each of these tests (Supplementary Figure 9).

1. Interpolating missing time periods within each NGA: We linearly interpolate PC1 by NGA to fill out the missing periods.

2. Weighting within each NGA depending on the sparsity of data within its neighborhood: For instance, say we observe an NGA at periods $t_{1}, t_{2}$, and $t_{3}$. We weigh the observation at $t_{2}$ by $\frac{\left(t_{2}-t_{1}\right)+\left(t_{3}-t_{2}\right)}{2}$ so 

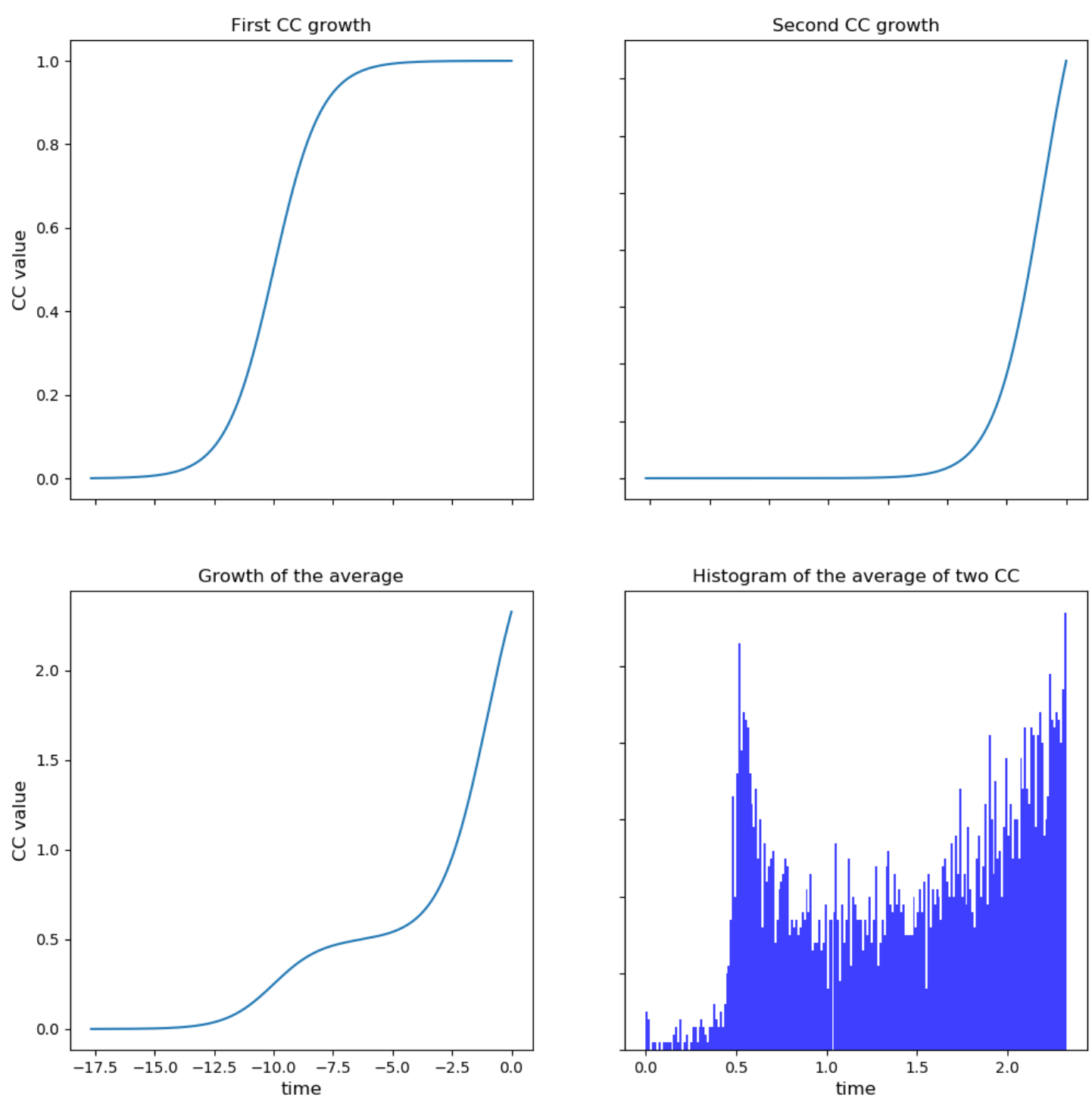

Supplementary Figure 7. The simulated logistic growth of two CCs ((a) and (b)), the growth of the average of the two (c), and the histogram of the average under uniform distribution.

that the observation in a sparse period counts more.

3. Use data points only from the NGAs that span both Gaussians: We take the NGAs that are classified as the first Gaussian in some periods, and the second Gaussian in other periods.

Supplementary Figure 9 exhibits the density plot with the main PC value and the normalized density as the two axes by linearly interpolating the main PC values for each NGA for missing periods, showing that the two peaks remain for all these cases. We found the two clusters of points, each corresponding 

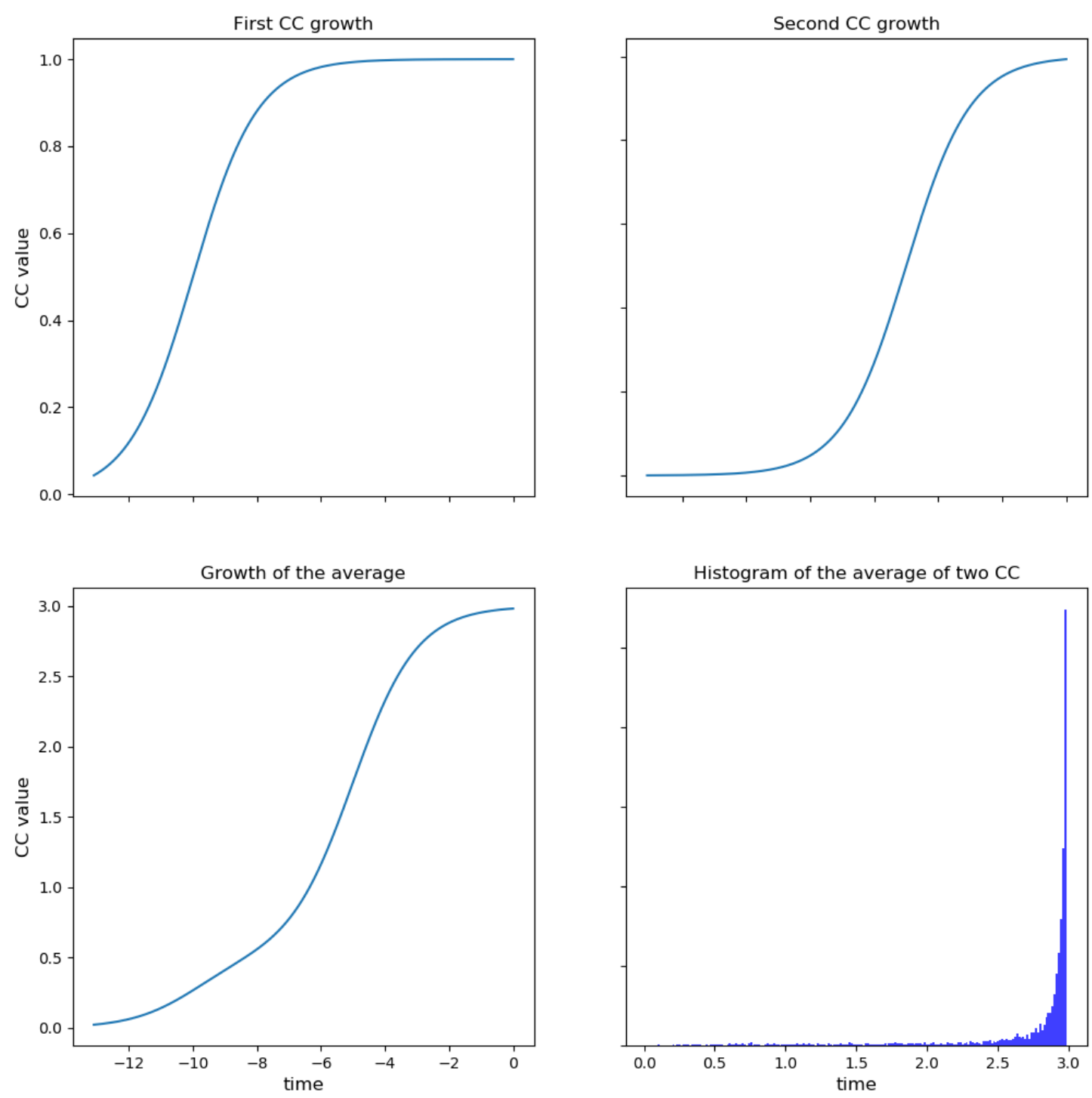

Supplementary Figure 8. The simulation of logistic growth CCs with different parameters.

to peaks in the bimodal curve after doing a scatter plot under each method, with time and the main PC value as the two axes. Also, we found that there were fast transitions from one (lower) cluster to the other (higher cluster).

\section{Plots of PC1 by Time}

In the main text we provide several plots showing PC1 scores by PC2 scores. Supplementary Figure 10 provides plots of PC1 scores through time for each NGA. Note that if each of these 30 sequences exhibited 


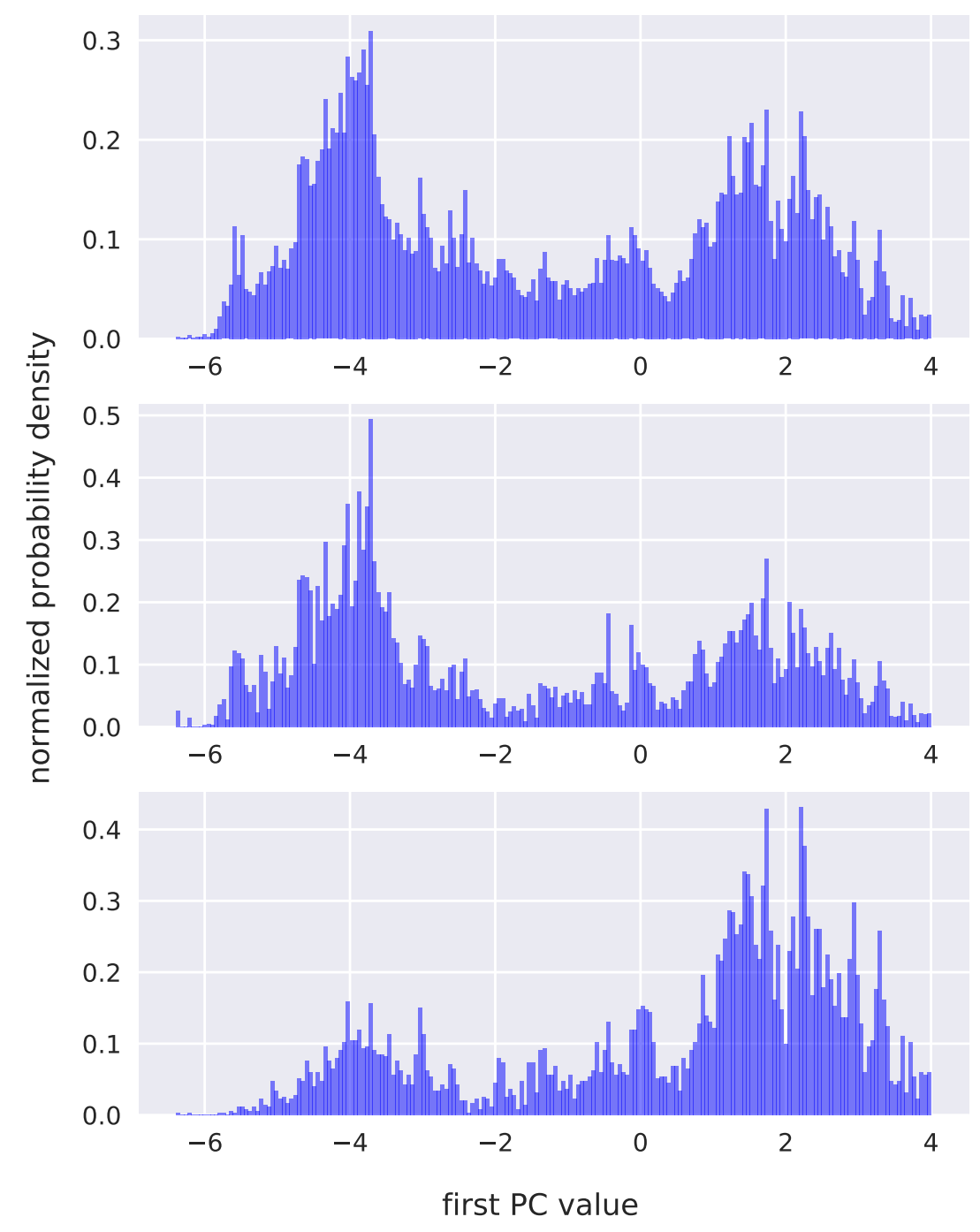

Supplementary Figure 9. Checking for undersampling bias in the data through interpolation of time. (Top) PC1 linearly interpolated for each NGA for missing periods. (Middle) Each observation weighted based on its relative sparsity with respect to each NGA, so that observations in sparse periods weigh more. (Bottom) Only data points from NGAs that span both Gaussians

a perfect positive correlation of its score on $\mathrm{PC} 1$ with its date, then we would be incorrect to maintain that the pattern-based correlational analysis undertaken $b^{2}{ }^{2}$ cannot stand in for the dynamics. However, this is clearly not the case. Moreover, even if this were true, we see that these individual sequences exhibit very different slopes and intercepts - a fact of substantive interest though not pursued in this article. Of course we should be wary of putting too much emphasis on such a correlational analysis since some of the values in these figures are interpolated. 

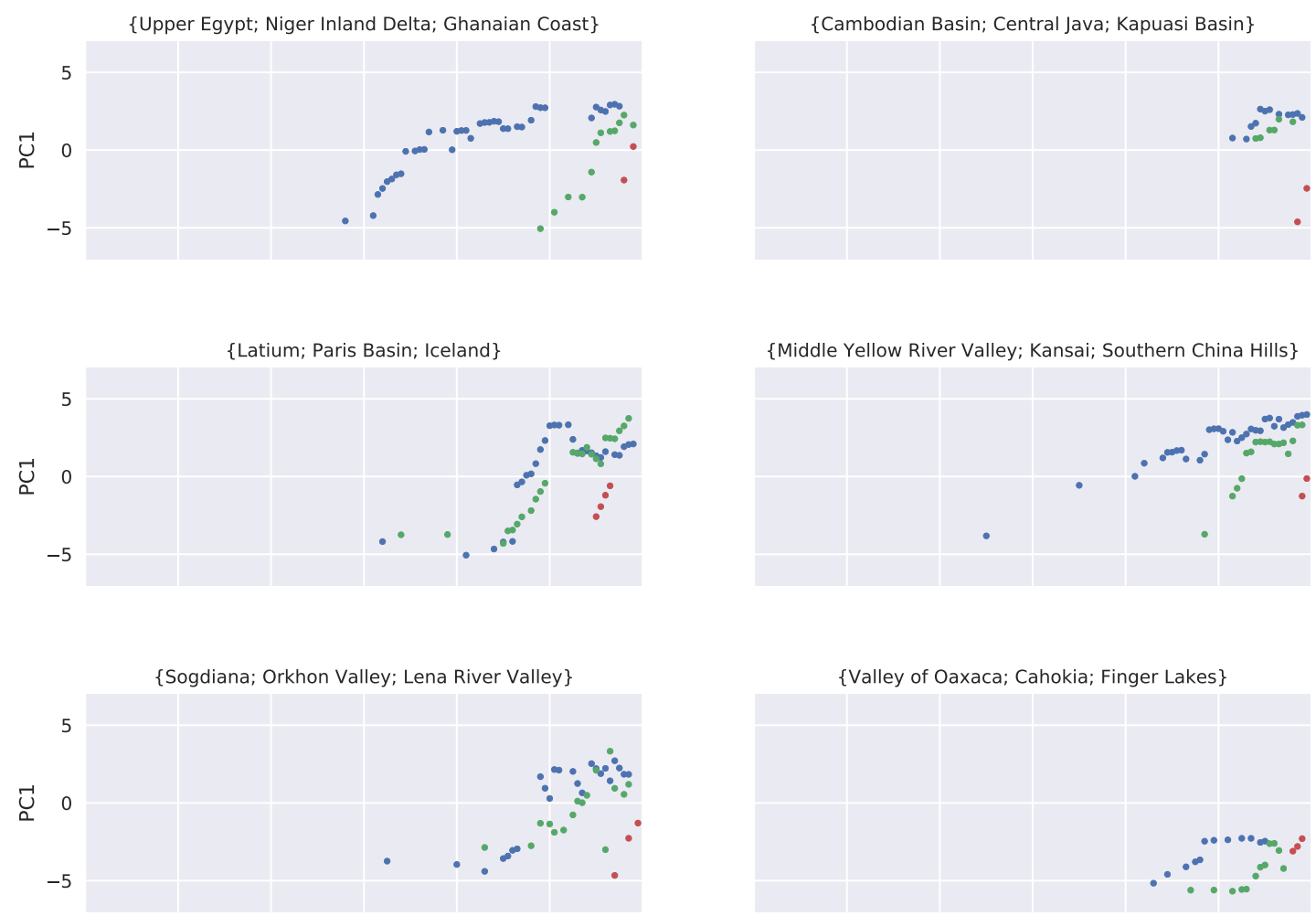

\{Valley of Oaxaca; Cahokia; Finger Lakes\}
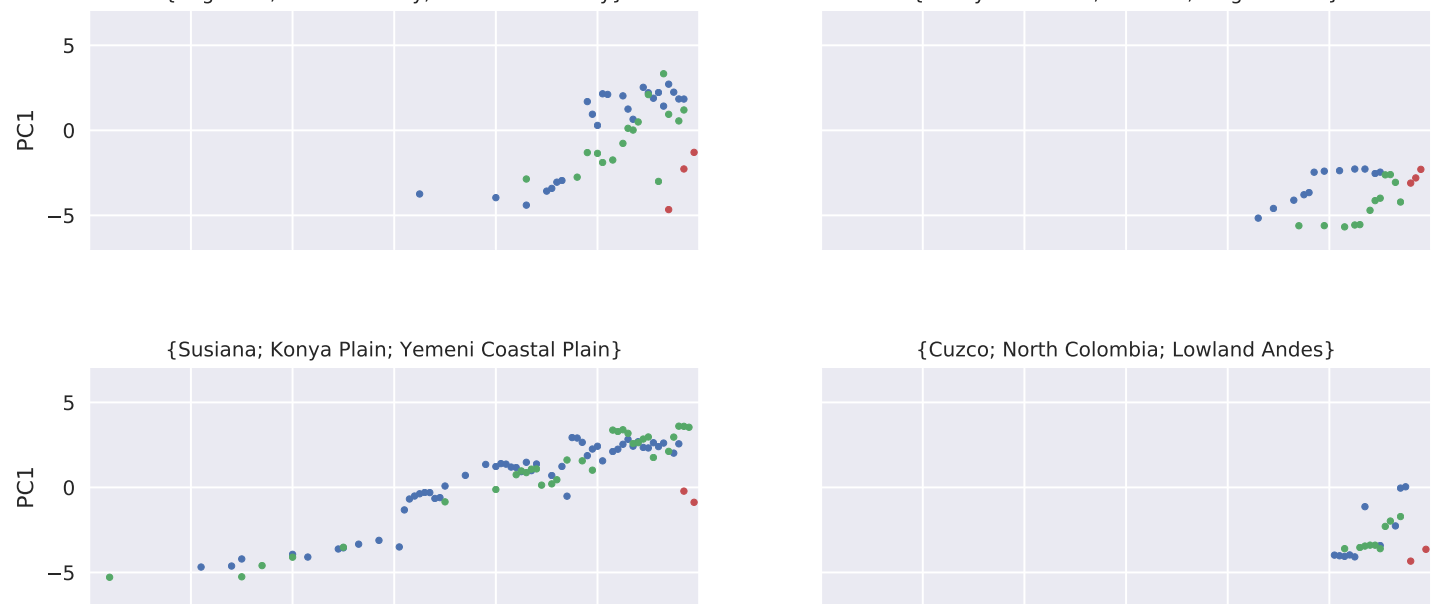

\{Cuzco; North Colombia; Lowland Andes\}
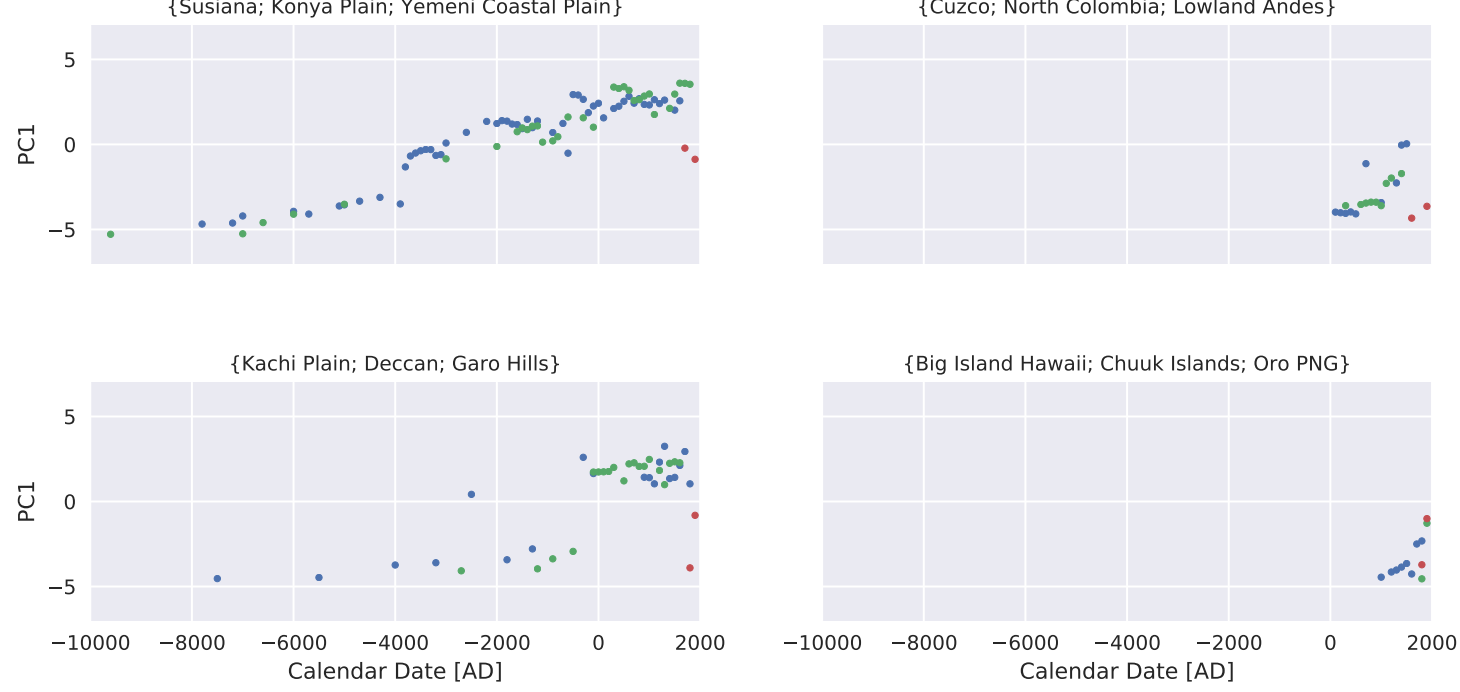

Supplementary Figure 10. PC1 as a function of time for 30 NGAs in 10 regions. Blue, green, and red points are, respectively, NGAs in each region with early, intermediate, and late onsets of centralization. The regions are (left column): Africa, Europe, Central Eurasia, Southwest Asia, South Asia; and (right column) Southeast Asia, East Asia, North America, South America, and Oceania-Australia. The PC1/19 value is the mean across 20 imputations. 


\section{References}

1. Turchin, P. et al. Seshat: The global history databank. Cliodynamics 6, 77-107 (2015).

2. Turchin, P. et al. Quantitative historical analysis uncovers a single dimension of complexity that structures global variation in human social organization. Proc. Natl. Acad. Sci. 115, E144-E151, DOI: 10.1073/pnas.1708800115 (2018). http://www.pnas.org/content/115/2/E144.full.pdf.

3. Whitehouse, H. et al. Complex societies precede moralizing gods throughout world history. Nature DOI: 10.1038/s41586-019-1043-4 (2019).

4. Turchin, P. Fitting dynamic regression models to seshat data. Cliodynamics 9, 25-58 (2018).

5. Turchin, P. et al. Evolutionary pathways to statehood: Old theories and new data. SocArXiv Prepr. DOI: 10.31235/osf.io/h7tr6 (2018).

6. Slingerland, E. et al. Historians respond to Whitehouse et al. (2019), "Complex societies precede moralizing gods throughout world history". PsyArXiv Prepr. DOI: 10.31234/osf.io/2amjz (2019).

7. Peregrine, P. N. Toward a theory of recurrent social formations. In Sabloff, J. A. \& Sabloff, P. L. W. (eds.) The Emergence of Premodern States: New Perspectives on the Development of Complex Societies, 271-295 (SFI Press, 2018). 